\title{
Rational design of Autotaxin inhibitors by structural evolution of endogenous modulators
}

Willem-Jan Keune, ${ }^{\dagger}$ Frances Potjewyd, ${ }^{\ddagger}$ Tatjana Heidebrecht, ${ }^{\dagger}$ Fernando Salgado-Polo, ${ }^{\dagger}$ Simon J. F. Macdonald," Lakshman Chelvarajan, "Ahmed Abdel Latif," Sony Soman," Andrew J. Morris, ${ }^{\#}$ Allan J. B. Watson, ${ }^{\ddagger}$ Craig Jamieson,,${ }^{\neq}{ }^{*}$ Anastassis Perrakis ${ }^{\dagger,}$

${ }^{\dagger}$ Division of Biochemistry, The Netherlands Cancer Institute, Plesmanlaan 121, 1066CX Amsterdam (NL)

${ }^{\ddagger}$ Department of Pure and Applied Chemistry, University of Strathclyde, 295 Cathedral Street, Glasgow, G1 1XL (UK)

"GlaxoSmithKline R\&D, Gunnels Wood Road, Stevenage, Herts SG1 2NY (UK)

\# Division of Cardiovascular Medicine and the Gill Heart Institute, Lexington Veterans Affairs Medical Center, Lexington KY 40536 (USA)

\section{Abstract}

Autotaxin produces the bioactive lipid lysophosphatidic acid (LPA), and is a drug target of considerable interest for numerous pathologies. We report the expedient, structure-guided evolution of weak physiological allosteric inhibitors (bile salts) into potent competitive Autotaxin inhibitors that do not interact with the catalytic site. Functional data confirms that our lead compound attenuates LPA mediated signalling in cells, and reduces LPA synthesis in vivo, providing a promising natural product derived scaffold for drug discovery. 


\section{Introduction}

Autotaxin (ATX, ENPP2) is responsible for the production of the bioactive lipid Iysophosphatidic acid (LPA) from lysophospholipids which exist extracellularly, primarily derived from lysophosphatidylcholine (LPC). ${ }^{1}$ The product of this process, LPA, is an agonist of six $\mathrm{G}$ protein-coupled receptors that drives a range of signaling pathways. ${ }^{2}$ This action produces a broad range of biological outcomes, and the pathophysiology of ATX has been associated with pulmonary fibrosis, tumor progression, cardiovascular disease, neuropathic pain, and cholestatic pruritus; these functions make the ATX-LPA signaling pathway an attractive prospect from the point of view of therapeutic intervention. ${ }^{3}$

The catalytic domain of the enzyme consists of an active site containing to zinc ions in proximity to a catalytic threonine. The endogenous ligand (LPC) binds in a shallow hydrophilic groove that is capable of accommodating both nucleotides as well as the glycerol motif of lysophospholipids. There also exists a relatively deep hydrophobic pocket which is responsible for binding of the lysophospholipid acyl chain. The catalytic domain in conjunction with the first of two somatomedin- $\beta$ (SMB) domains, constitutes a tunnel adjacent to the active site. ${ }^{4-5}$ Recently, we reported that ATX produced in mammalian cells contains steroids bound within the tunnel region. During that study, we determined the structures of natural endogenous steroids bound to the ATX tunnel, including that of tauroursodeoxycholic acid (TUDCA, 1, Figure 1), a micromolar inhibitor of LPA production. We demonstrated that binding of bile salts, allosterically modulates ATX activity through non-competitive inhibition, likely having physiologically important implications for the function of the ATX-mediated signaling through LPA receptors in a number of pathophysiological contexts. ${ }^{6}$

The majority of small molecule inhibitors of ATX developed to date emulate the lipid-like structure of the native ligand and bind in the orthosteric site (e.g. 3, Figure 1). By contrast, a recent series of indole-based ATX inhibitors ${ }^{7}$ and the lead compound of an independent discovery program ${ }^{8}$ are reported to occupy the hydrophobic pocket and tunnel, without 
interacting with the hydrophilic groove or the active site, acting as allosteric inhibitors, similar to natural bile salts.

In this manuscript, we formulate a design hypothesis based on structural knowledge of the binding mode of the partial non-competitive allosteric inhibitor bile salts, and competitive orthosteric inhibitors. ${ }^{9}$ Specifically, we used the structure of compound 1 bound to ATX (Figure 1) which we recently determined binding in the allosteric tunnel region, ${ }^{6}$ and the structure of a compound from the represented by compound 2 (PF-8380), ${ }^{10}$ which we had determined in complex with ATX, showing the binding of this chemotype in the orthosteric site.

From consideration of these binding modes we inferred that the tunnel-binding steroid skeleton could be merged with the lipophilic pocket-binding "tail" of compound 2 . While the "tail" of compound $\mathbf{2}$ is in isolation ineffective,${ }^{11}$ as is the steroid group alone,${ }^{6}$ we reasoned the hybridized structures may be potent inhibitors of ATX. 


\section{Results and Discussion}

We crystallized an alcohol analogue (3, Figure 2) of compound 2, which, owing to its significantly higher aqueous solubility, proved more amenable to co-crystallization compared to the parent compound, 2. We determined the crystal structure of $\mathbf{3}$ bound to ATX (Figure 1 A-C) to $2.1 \AA$ resolution, showing that the 3,5-dichlorobenzyl moiety of 3 binds in the lipid binding pocket, consistent with a recent report of the binding mode of this series. ${ }^{12}$ Superposing this structure of $\mathbf{3}$, with that of 1 in complex with ATX $^{6}$ (Figure 1 D-F) enabled the design of a highly chemically tractable hybrid series through the union of the two binding motifs (Figure 2).

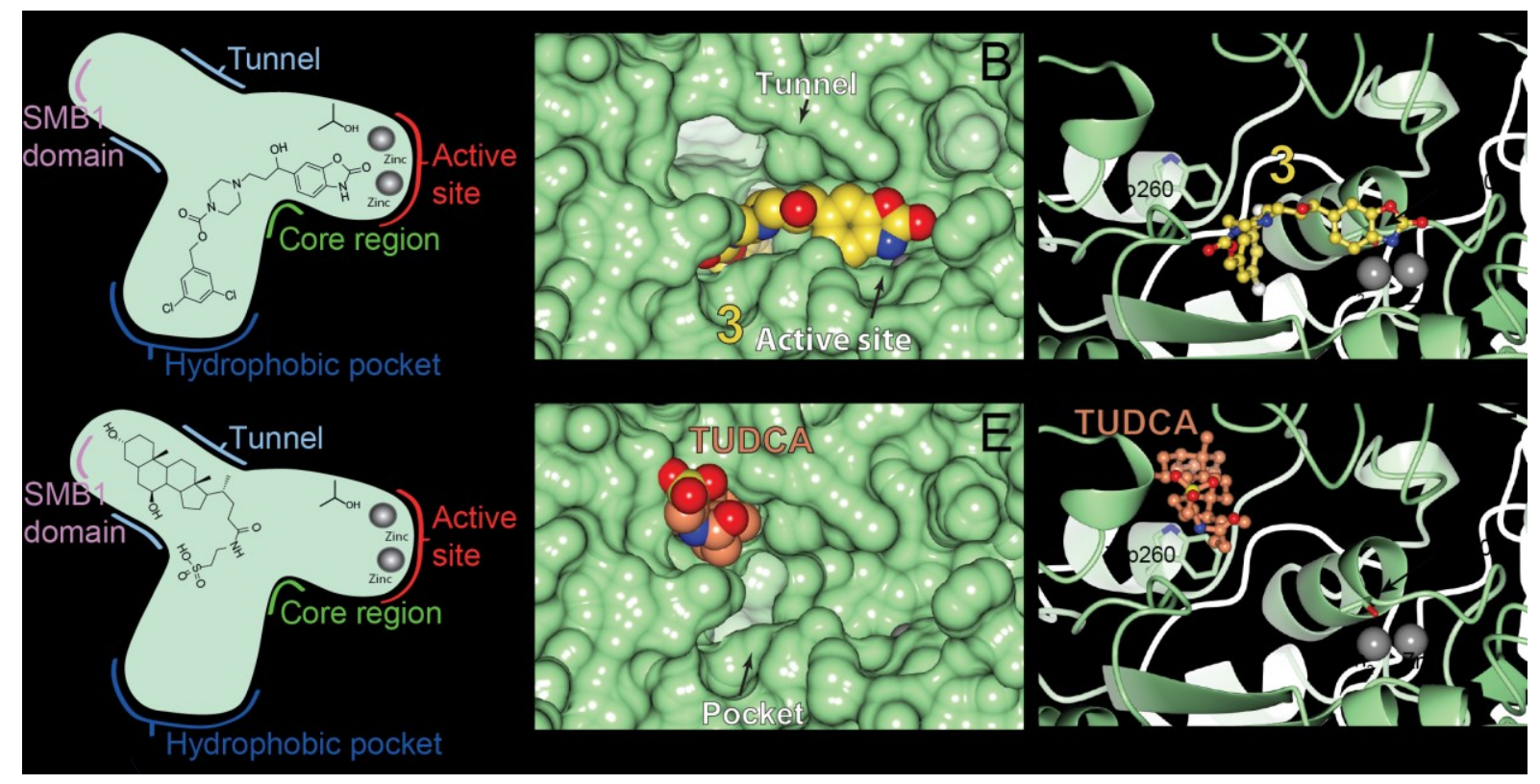

Figure 1. Crystal structure of 3 and 1 binding to the hydrophobic pocket and the tunnel of ATX, respectively. (A-C) show that 3 binds to the hydrophobic pocket. Compound 3 is racemic and the $(R)$ enantiomer is resolved through co-crystallography; (D-F) show that 1 binds to the tunnel. 


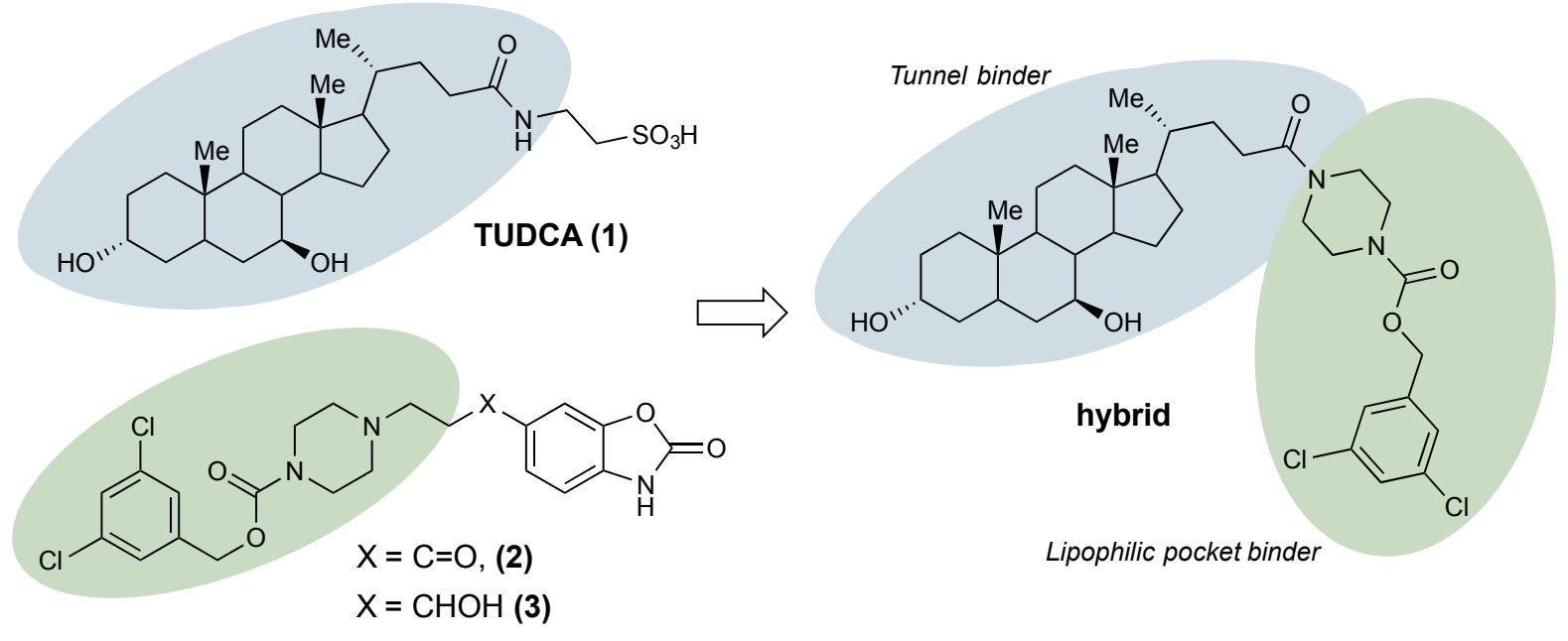

Figure 2. Overarching design hypothesis

These compounds could be rapidly prepared through HATU-mediated amide bond formation between ursodeoxycholic acid (UDCA) and appropriate amine fragments (Scheme 1). Compound 11 was accessed through reduction of the amide to give the corresponding amine derivative. The requisite amine components could be prepared through carbamate formation on the mono-protected diamine, followed by deprotection to furnish the free amine as represented by the example shown in Scheme 2.

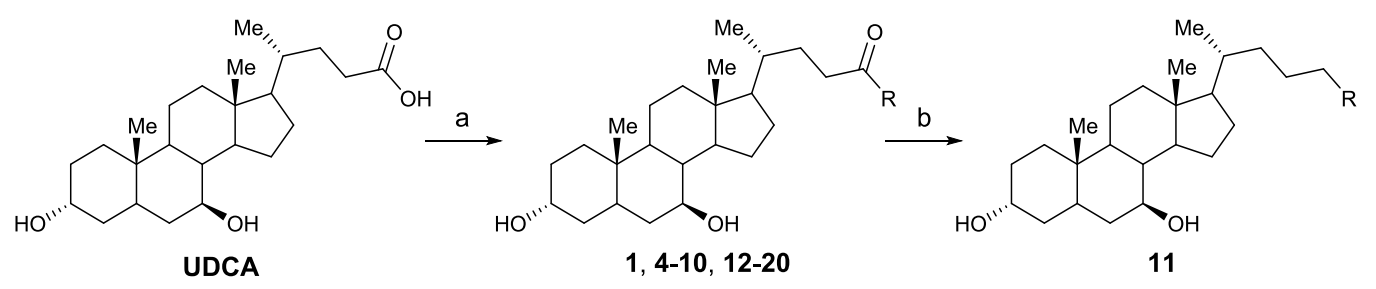

Scheme 1. Conditions (a) amine (0.9 equiv), HATU (1.0 equiv), DIPEA (4.0 equiv), DMF, rt; (b) $\mathrm{BH}_{3} \cdot \mathrm{THF}$ (10 equiv), THF, $60{ }^{\circ} \mathrm{C}$.<smiles>CC(C)(C)OC(=O)N1CCN(C(=O)OCc2cc(Cl)cc(Cl)c2)CC1</smiles>

21

23

Scheme 2. (a) 1,1'-carbonyldiimidazole (1.1 equiv), 3,5-dichlorobenzylalcohol (1.1 equiv), amine (1.0 equiv), DMF, rt; (b) Trifluoroacetic acid/ $\mathrm{CH}_{2} \mathrm{Cl}_{2}$ (1:1). 
To our delight, the first hybrid compound prepared (4, Table 1 ) showed approximately an 80 fold increase in potency relative to the progenitor bile acid $(0.129 \mu \mathrm{M}$ compared to $10 \mu \mathrm{M}$, determined through measurement of inhibition of LPC hydrolysis), confirming that the concept of maintaining the steroid moiety while also targeting the hydrophobic pocket of ATX had indeed increased inhibitor potency. In agreement with this hypothesis, compounds without chlorine substituents (9-10) or lacking a hydrophobic anchor entirely (8) in this initial set of analogues, results in no detectable inhibitory activity. These findings are consistent with SAR previously established for the hydrophobic pocket, all of which indicate a degree of lipophilicity in this region is required for adequate levels of ATX-inhibitory activity. ${ }^{10-13}$

In this initial survey of SAR, we also sought to examine the length and nature of the linker (57). While the piperazine unit in $\mathbf{4}$ was optimal, linear systems exhibit only slightly lower potency (5-6); however, a very short spacer (7) resulted in no detectable inhibitory activity. Compound 11, lacking the amide carbonyl of 4 , had five-fold lower potency. 
Table 1. Evaluation of hybrid compounds.
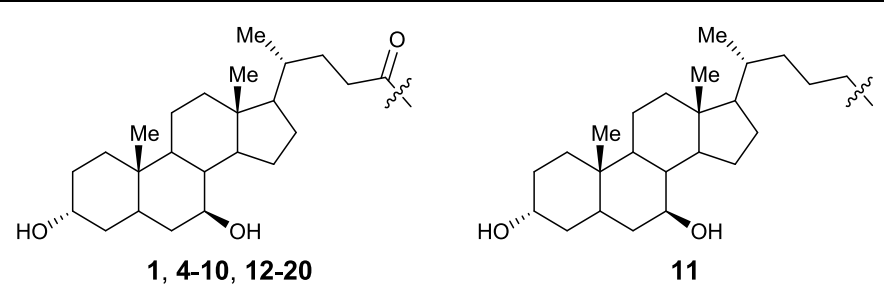

\begin{tabular}{|c|c|c|c|c|c|c|c|}
\hline Compound & $\begin{array}{l}I_{50} \\
(\mu \mathrm{M})\end{array}$ & MW & $\log D^{b}$ & Compound & $\begin{array}{l}I_{50} \\
(\mu M)\end{array}$ & MW & $\log D^{b}$ \\
\hline 1 & 10.3 & 500 & -0.2 & $\begin{array}{l}-\xi-\mathrm{NH} \\
12\end{array}$ & 2.5 & 692 & 6.4 \\
\hline & 0.129 & 664 & 6.0 & & 0.100 & 708 & 5.2 \\
\hline & 0.202 & 638 & 5.7 & & $2.10^{a}$ & 675 & 5.4 \\
\hline$-\xi-\mathrm{NH}$ & 0.563 & 652 & 5.8 & 15 & 1.00 & 650 & 5.8 \\
\hline $\begin{array}{l}-\xi-N H \\
7\end{array}$ & $>30$ & 551 & 6.1 & 16 & 0.208 & 650 & 5.8 \\
\hline $\begin{array}{l}-\xi-N H \\
8\end{array}$ & $>30$ & 435 & 0.6 & $\begin{array}{l}\text { P. } \\
17\end{array}$ & 0.020 & 664 & 5.9 \\
\hline 9 & $>30$ & 569 & 4.5 & & 0.717 & 664 & 5.9 \\
\hline 10 & $>30$ & 595 & 4.8 & & 0.313 & 664 & 5.9 \\
\hline & 0.814 & 650 & 6.6 & & 0.500 & 664 & 5.9 \\
\hline
\end{tabular}

[a] Epimeric mixture; [b] calculated at $\mathrm{pH} 7.4$ using JChem for Excel. ${ }^{14}$ 

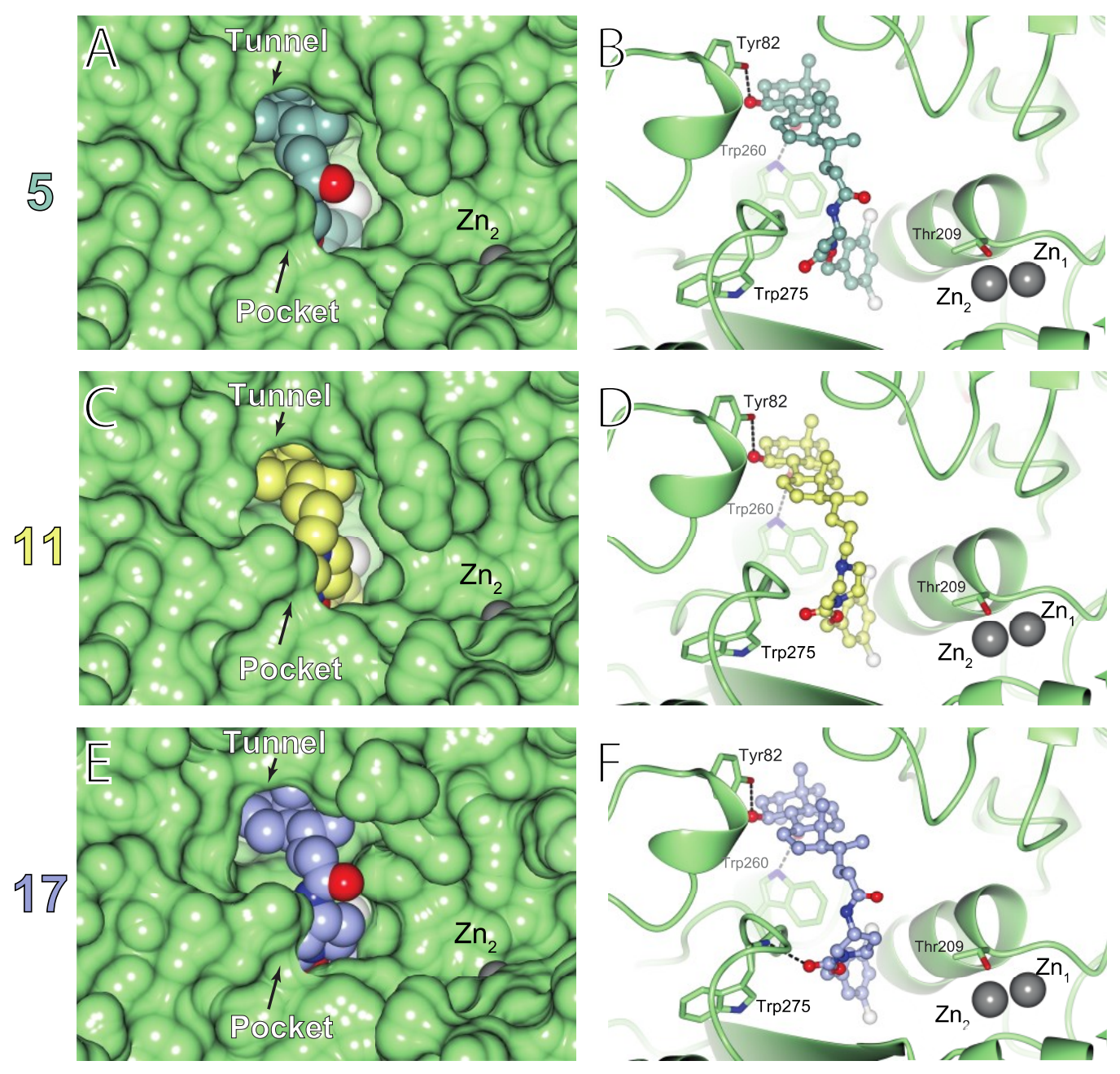

Figure 3. Crystal structure of ATX bound to 5, 11 and 17. The view and representation is similar to Figure 1. 

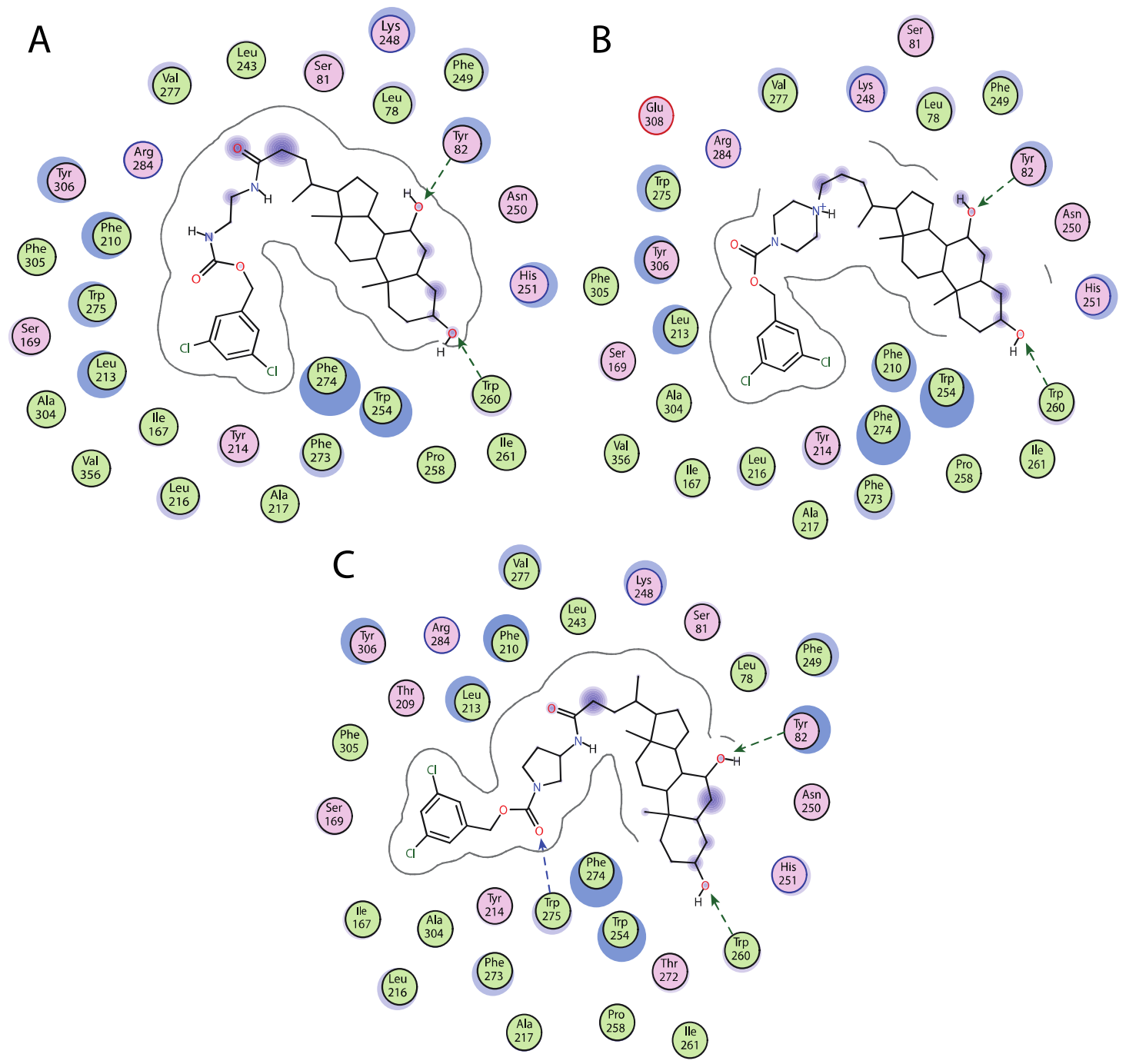

Figure 4. Two-dimensional projection depicting the interactions between the three ligands 5 (A), 11 (B), 17 (C) and ATX, highlighting hydrogen bonds with Trp275, Tyr82 and Trp260 of ATX (arrows).

To further rationalize the SAR data obtained and confirm our proposed binding mode hypothesis, we determined the crystal structure of ATX bound to 5 and 11 to $2.4 \AA$ and $2.1 \AA$ resolution, respectively (Figure $3 A-D$ and Figure $4 A$ and B). These structures fully support our original design hypothesis and demonstrate that the hydrophobic anchor, the 3,5dichlorobenzyl group, occupies essentially the same position in the hydrophobic pocket in the structures of $\mathbf{3}, \mathbf{5}$, and $\mathbf{1 1}$. Notably, the steroid moiety also remains in a similar, albeit not identical binding orientation between structures, when comparing the structures of $\mathbf{1 , 5}$, and 11, and is held in position by several interactions including two hydrogen bonds, with the 
Tyr82 and Trp260 side chains. The piperazine linker unit, however, adopts a significantly different conformation in 11 compared to 3 , projecting towards the tunnel, instead of the catalytic site; the linear linker in $\mathbf{5}$ follows a similar vector to that of the piperazine in $\mathbf{1 1 .}$ Interestingly, the linker region in both structures does not exhibit well defined electron density compared to the other groups, suggesting a certain degree of structural flexibility in the region, with the linker potentially adopting several conformations. Accordingly, these structures suggest that the bile salt steroid moiety and the dichlorobenzyl group act as anchors in the tunnel and the pocket, respectively, and optimization of binding should subsequently focus on the linker region to provide a conformation which may lead to optimal binding. Based on this, the 3,5-dichlorobenzyl group was retained in subsequent analogues prepared, and our focus turned to tuning the spacer region of our nascent series.

Several isosteric cores were evaluated (12-20, Table 1) with a range of ATX inhibitory activity noted. Aminomethyl piperidine systems were tolerated with a preference for the exocyclic carbamate (cf. 12-13), and more constrained systems such as 14 led to a 10-fold reduction in activity. Other isosteres such as azetidinyl and 3-amino pyrrolidine (15-20) showed comparable levels of potency relative to the progenitor compound 4. A notable exception is evidenced by compound 17, displaying an $\mathrm{IC}_{50}$ of $20 \mathrm{nM}$ against ATX mediated production of LPA, which compares favorably to $2\left(\mathrm{IC}_{50}=3 \mathrm{nM}^{10}\right)$. Additionally, This represents an over 500-fold improvement in activity compared to the parent bile acid, and demonstrates the utility of a structure based design approach in expediently optimizing a weak endogenous modulator of ATX activity towards a highly potent lead candidate.

The crystal structure of 17 bound to ATX (Figure $3 \mathrm{E}$ and F, Figure $4 \mathrm{C}$ ) was determined to $1.95 \AA$ resolution, and illustrates that similar to the structures of 5 and $\mathbf{1 1}$, the $3,5-$ dichlorobenzyl and the steroid groups again act as anchors in the pocket and the tunnel, respectively, making several contacts with ATX, and occupying essentially the same positions as in complexes of 5 and 11. The two hydrogen bonds between ATX and the steroid moiety of hybrid inhibitors remain the same in the complex of ATX with 17 (Figure 4 
C) as for the complexes with 5 and 11; however, the positioning of the new linker allows the formation of an additional hydrogen bond between the urethane carbonyl and the main chain amide of Trp275. This observation could account in part for the increased potency of 17. In addition, the electron density for the linker region is better defined in this structure, indicating a more restricted spatial distribution of the linker. The linker of $\mathbf{1 7}$ also has a better fit to the ATX surface (compare Figure $3 \mathrm{E}$ to $3 \mathrm{~A}, \mathrm{C}$ ). Collectively, these structural observations account for the superior activity of 17 .

To further characterize the potency and mechanism of inhibition, we examined inhibition of LPC hydrolysis by 17 in greater depth, based on measurement of a single LPA species, (18:1, referring to an 18 carbon chain with a single degree of unsaturation). As anticipated, 17 was shown to be a competitive inhibitor of LPC hydrolysis (Figure 5) with a $K_{i}$ of 0.006 $\mu \mathrm{M}$. This contrasts with the progenitor steroid 1 which is a weak non-competitive inhibitor of LPC hydrolysis. Accordingly, by targeting the hydrophobic pocket, we have switched the mechanism of action in this emerging lead series compared to the endogenous modulator 1 .
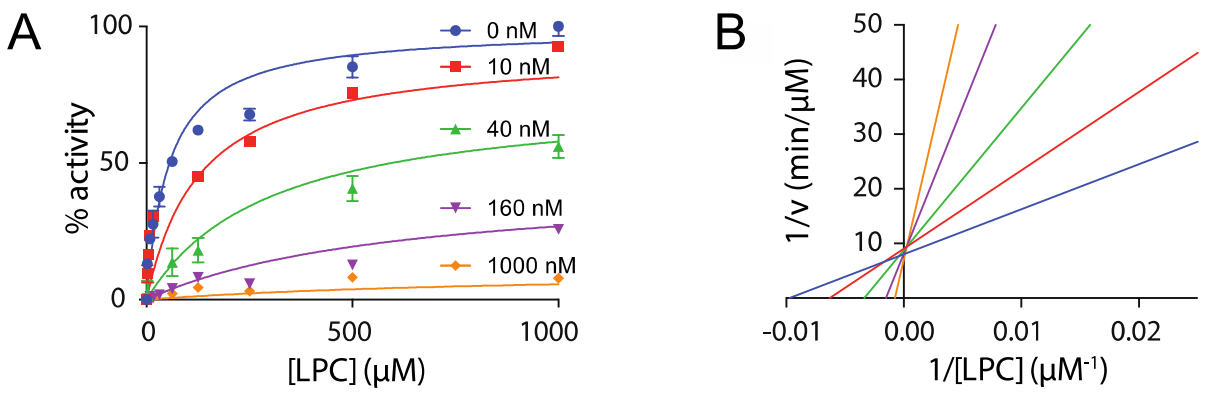

Figure 5. 17 acts as a competitive inhibitor of LPC hydrolysis. (A) ATX lysoPLD activity with no inhibitor (blue symbols) and with four concentrations of 17 as a function of LPC (18:1) substrate concentrations; the lines represent the non-linear regression fit of all data $(B)$ a simulated Lineweaver-Burk plot, fitting the velocity for each experiment separately, graphically demonstrating that $\mathbf{1 7}$ acts as a competitive inhibitor of LPC hydrolysis with a $\mathrm{K}_{\mathrm{i}}$ of $6 \pm 2 \mathrm{nM}$.

Having developed a novel tool compound with known mechanism of action, we subsequently sought to demonstrate that 17 inhibits LPA-mediated signaling in cancer cells, by examining its ability to block phosphorylation of the AKT kinase; consistently, concentrations of above $0.1 \mu \mathrm{M}$ of 17 decreases the level of pAKT, as detected by Western 
blot analysis (Figure $6 \mathrm{~A}$ ), suggesting that compound $\mathbf{1 7}$ is a viable tool in a physiologically relevant context.

Lastly, in order to examine inhibition of ATX in vivo, 17, and $\mathbf{2}$ as a positive control, were administered to mice (10 mg/kg, i.p.) and the levels of eight abundant LPA species were summed and determined by mass spectrometry. ${ }^{15}$ The in vivo activity of 17 was similar to that of 2, reducing LPA levels to about half to that of control mice injected with vehicle alone (Figure $6 \mathrm{~B}$ ). Although determination of in vivo half-life was not possible in this experiment, the data indicates that compound 17 must have sufficient degree of stability in order to be able to modulate LPA levels 8 hours following i.p. administration. This highly encouraging data indicates robust target engagement in an in vivo setting, thus validating our emerging lead series as promising tools to further prosecute the biology of Autotaxin in a variety of disease states.

A

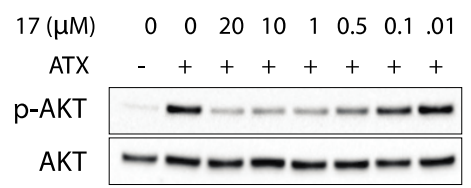

B

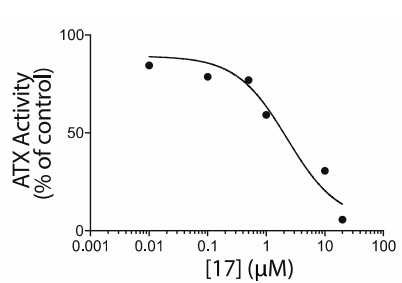

C

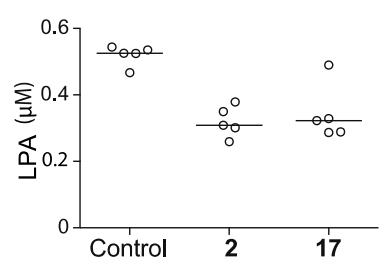

Figure 6. (A) Western-blot showing inhibition of ATX by 17 decreases Ser473 phosphorylation of AKT in BJEH cells. (B) Graphical depiction of Western-blot using densitometry. (C) LPA levels measured for the injection of control, 2 and 17, showing the levels of LPA in the individual animals (circles) and the median value (horizontal line); Student's unpaired two tailed T-test suggests the difference of $\mathbf{2}$ and $\mathbf{1 7}$ to the control is significant $(p<0.01)$.

In summary, we report the development of an endogenous steroid modulator of Autotaxin function towards a highly potent hybrid lead series with significant levels of activity in vivo. The optimization trajectory was significantly expedited through application of a structurebased design approach, leading to the rapid identification of compound $\mathbf{1 7}$ which could be differentiated from the progenitor steroid both in terms of significantly enhanced potency and mechanism of action (competitive vs non-competitive inhibition of LPA hydrolysis). The 
structural data generated confirmed the original design hypothesis, and support the observed mechanism of action for the series lead, compound 17. Inhibition of ATX activity by our lead compound in cells and in a pilot experiment in mice, suggest that these new entities will serve as valuable tools in further understanding the pathophysiological roles of Autotaxin. 


\section{Experimental}

Chemistry. General. All reagents and solvents were obtained from commercial suppliers and were used without further purification unless otherwise stated. Purification was carried out according to standard laboratory methods. Reactions were carried out using conventional glassware room temperature was generally $18^{\circ} \mathrm{C}$. Reactions were carried out at elevated temperatures using a temperature-regulated hotplate/stirrer. Thin layer chromatography was carried out using Merck silica plates coated with fluorescent indicator UV254. These were analyzed under $254 \mathrm{~nm}$ UV light or developed using potassium permanganate solution. Normal phase flash chromatography was carried out using ZEOprep 60 HYD 40-63 $\mu \mathrm{m}$ silica gel. Fourier Transformed Infra-Red (FTIR) spectra were obtained on a Shimadzu IRAffinity-1 machine. ${ }^{1} \mathrm{H}$ and ${ }^{13} \mathrm{C}$, NMR spectra were obtained on a Bruker AV 400 at $400 \mathrm{MHz}$ and $101 \mathrm{MHz}$, respectively and a Bruker AVIIIHD500 at $500 \mathrm{MHz}$ and 126 $\mathrm{MHz}$, respectively. Chemical shifts are reported in ppm and coupling constants are reported in $\mathrm{Hz}$ with $\mathrm{CDCl}_{3}$ referenced at $7.26\left({ }^{1} \mathrm{H}\right)$ and $77.1 \mathrm{ppm}\left({ }^{13} \mathrm{C}\right)$ and DMSO- $d_{6}$ referenced at $2.50\left({ }^{1} \mathrm{H}\right)$ and $39.52 \mathrm{ppm}\left({ }^{13} \mathrm{C}\right)$. Compound purity was determined by LCMS and NMR analysis, and all compounds were $\geq 95 \%$ purity. High-resolution mass spectra were obtained through analysis at the EPSRC UK National Mass Spectrometry Facility at Swansea University or in the School of Chemistry at the University of Glasgow, UK.

General Procedure A: Carbamate Formation. For example, for the preparation of 1-(tertbutyl) 4-(3,5-dichlorobenzyl) piperazine-1,4-dicarboxylate, 21. ${ }^{11}$ To a round bottom flask was added (3,5-dichlorophenyl)methanol (1.04 g, $5.91 \mathrm{mmol}, \quad 1.10$ equiv), 1,1'carbonyldiimidazole $(0.96 \mathrm{~g}, 5.91 \mathrm{mmol}, 1.10$ equiv) in DMF ( $5 \mathrm{~mL})$ and stirred for $30 \mathrm{~min}$ at room temperature. To the reaction mixture was added tert-butyl piperazine-1-carboxylate (1.00 g, $5.37 \mathrm{mmol}, 1.00$ equiv) and stirred for $30 \mathrm{~min}$ at room temperature. The reaction was diluted with $\mathrm{H}_{2} \mathrm{O}(10 \mathrm{~mL})$ and the precipitate filtered under vacuum with $\mathrm{H}_{2} \mathrm{O}$, and dried in a dessicator to afford the desired product as a white solid (1.87 $\mathrm{g}, 90 \%)$. Umax (neat): 
2847, 1698, 1678, $1418 \mathrm{~cm}^{-1} .{ }^{1} \mathrm{H}$ NMR $\left(\mathrm{CDCl}_{3}, 400 \mathrm{MHz}\right): \delta 7.34(\mathrm{t}, J=1.9 \mathrm{~Hz}, 1 \mathrm{H}), 7.25(\mathrm{~d}$, $J=1.91 \mathrm{~Hz}, 2 \mathrm{H}), 5.10(\mathrm{~s}, 2 \mathrm{H}), 3.55-3.48(\mathrm{~m}, 4 \mathrm{H}), 3.48-3.42(\mathrm{~m}, 4 \mathrm{H}), 1.49(\mathrm{~s}, 9 \mathrm{H}) .{ }^{13} \mathrm{C}$ NMR $\left(\mathrm{CDCl}_{3}, 101 \mathrm{MHz}\right): \delta 154.8,154.7,140.0,153.3,128.4,126.3,80.4,65.8,43.9,28.5 .1 \times \mathrm{C}$ not observed. HRMS: exact mass calculated for $\left[\mathrm{M}+\mathrm{NH}_{4}\right]^{+}\left(\mathrm{C}_{17} \mathrm{H}_{26} \mathrm{Cl}_{2} \mathrm{~N}_{3} \mathrm{O}_{4}\right)$ requires $\mathrm{m} / \mathrm{z}$ 406.1295, found $\mathrm{m} / \mathrm{z} 406.1293$.

General Procedure B: N-Benzyl Carbamate Formation. For example, for the preparation of benzyl tert-butyl ethane-1,2-diyldicarbamate, 22. ${ }^{11}$ To a round bottom flask was added tert-butyl 3-methylpiperazine-1-carboxylate $\quad(100 \mathrm{mg}, \quad 0.50 \mathrm{mmol}, \quad 1.00$ equiv), $\mathrm{N}$ (benzyloxycarbonyloxy)succinimide (137 mg, $0.55 \mathrm{mmol}, 1.10$ equiv) and $\mathrm{NaHCO}_{3}(66.4 \mathrm{mg}$, $0.75 \mathrm{mmol}, 1.40$ equiv), in THF: $\mathrm{H}_{2} \mathrm{O}(1 \mathrm{~mL}: 1 \mathrm{~mL})$ and stirred for $16 \mathrm{~h}$ at room temperature. The reaction mixture was diluted with $\mathrm{H}_{2} \mathrm{O}(20 \mathrm{~mL})$ and the organics were extracted with ethyl acetate $(2 \times 20 \mathrm{~mL})$, dried with a hydrophobic frit and concentrated in vacuo to afford the desired product as a clear oil (154 mg, 89\%). Umax (neat): 3314, 2977, 2931, 1689, 1546 $\mathrm{cm}^{-1} .{ }^{1} \mathrm{H}$ NMR $\left(\mathrm{CDCl}_{3}, 400 \mathrm{MHz}\right): \delta$ 7.39-7.28 (m, 5H), $5.29(\mathrm{~s}, 1 \mathrm{H}), 5.09(\mathrm{~s}, 2 \mathrm{H}), 4.94(\mathrm{~s}$, 1H), 3.32-3.15 (m, 4H), $1.42(\mathrm{~s}, 9 \mathrm{H}) .{ }^{13} \mathrm{C} \mathrm{NMR}\left(\mathrm{CDCl}_{3}, 101 \mathrm{MHz}\right): \delta 156.3,155.9,136.0$, 128.0, 127.63, 127.60, 79.1, 66.2, 41.0, 40.1, 27.9. HRMS: exact mass calculated for $[\mathrm{M}+\mathrm{H}]^{+}$ $\left(\mathrm{C}_{15} \mathrm{H}_{23} \mathrm{~N}_{2} \mathrm{O}_{4}\right)$ requires $295.1652 \mathrm{~m} / \mathrm{z}$, found $295.1654 \mathrm{~m} / \mathrm{z}$.

General Procedure C: $\mathbf{N}$-Boc deprotection. For example, for the preparation of 3,5dichlorobenzyl piperazine-1-carboxylate, 23. ${ }^{11}$ To a round bottom flask was added 1-(tertbutyl) 4-(3,5-dichlorobenzyl)piperazine-1,4-dicarboxylate $(1.87 \mathrm{~g}, 4.80 \mathrm{mmol})$, a mixture of trifluoroacetic acid: $\mathrm{CH}_{2} \mathrm{Cl}_{2}(1 \mathrm{~mL}: 1 \mathrm{~mL})$ and the reaction stirred at room temperature for $2 \mathrm{~h}$. The reaction mixture was concentrated in vacuo and purified by strong cation exchange eluting with $2 \mathrm{M} \mathrm{NH}_{3}$ in $\mathrm{MeOH}$ to afford the desired product as a white solid $(0.53 \mathrm{~g}, 60 \%)$. Consistent with previously reported spectral analysis. Umax (neat): 2918, 2849, 2494, 1670, 1425, $1128 \mathrm{~cm}^{-1} .{ }^{1} \mathrm{H}$ NMR $\left(\mathrm{CDCl}_{3}, 400 \mathrm{MHz},\right): \delta 7.26(\mathrm{t}, J=1.7 \mathrm{~Hz}, 1 \mathrm{H}), 7.20(\mathrm{~d}, J=1.8 \mathrm{~Hz}$, 
2H), $5.04(\mathrm{~s}, 2 \mathrm{H}), 3.51-3.45(\mathrm{~m}, 4 \mathrm{H}), 2.88-2.81(\mathrm{~m}, 4 \mathrm{H}), 2.02(\mathrm{~s}, 1 \mathrm{H}) .{ }^{13} \mathrm{C} \mathrm{NMR}\left(\mathrm{CDCl}_{3}, 101\right.$ MHz): $\delta 154.7,140.1,135.1,128.2,126.1,65.6,45.5,44.4$. HRMS: exact mass calculated for $[\mathrm{M}+\mathrm{H}]^{+}\left(\mathrm{C}_{12} \mathrm{H}_{15} \mathrm{Cl}_{2} \mathrm{~N}_{2} \mathrm{O}_{2}\right)$ requires $\mathrm{m} / \mathrm{z} 289.0505$, found $\mathrm{m} / \mathrm{z} 289.0503$.

General Procedure D: HATU amidation. For example, for the preparation of, 3,5dichlorobenzyl-4-((4R)-4-((3R,7S,10S,13R)-3,7-dihydroxy-10,13-dimethylhexadecahydro$1 \mathrm{H}$-cyclopenta[a]phenanthren-17-yl)pentanoyl)piperazine-1-carboxylate, 4 . To a round bottom flask was added ursodeoxycholic acid (100 mg, $0.26 \mathrm{mmol}, 1.1$ equiv) in DMF (1 mL), $\mathrm{N}, \mathrm{N}$-diisopropylethylamine $(180 \mu \mathrm{L}, 1.02 \mathrm{mmol}, 4.0$ equiv $)$ and then 1 [bis(dimethylamino)methylene]-1H-1,2,3-triazolo[4,5-b]pyridinium 3-oxide hexafluorophosphate (HATU, $97 \mathrm{mg}, 0.26 \mathrm{mmol}, 1.1$ equiv). After stirring at room temperature for $15 \mathrm{~min}$ 3,5-dichlorobenzyl piperazine-1-carboxylate $(67 \mathrm{mg}, 0.23 \mathrm{mmol}, 1.0$ equiv) was added and stirred for $16 \mathrm{~h}$ at room temperature. The reaction mixture was diluted with $\mathrm{H}_{2} \mathrm{O}(20 \mathrm{~mL})$ and the resulting precipitate filtered under vacuum. The crude material was purified by column chromatography on silica $(0-10 \% \mathrm{MeOH}$ in ethyl acetate) to afford the desired product as a clear solid (87 mg, 51\%). Umax (neat): 3496, 2931, 2866, 1708, 1637, 1421, $1231 \mathrm{~cm}^{-1} .{ }^{1} \mathrm{H}$ NMR $\left(\mathrm{CDCl}_{3}, 500 \mathrm{MHz}\right): \delta$ 7.32-7.30 (m, 1H), 7.24-7.21 (m, 2H), $5.08(\mathrm{~s}$, 2H), 3.65-3.44 (m, 9H), 2.42-2.32 (m, 1H), 2.28-2.18 (m, 1H), 2.02-1.96 (m, 1H), 1.96-1.85 $(\mathrm{m}, 1 \mathrm{H}), 1.85-1.74(\mathrm{~m}, 4 \mathrm{H}), 1.74-1.63(\mathrm{~m}, 3 \mathrm{H}), 1.63-1.55(\mathrm{~m}, 2 \mathrm{H}), 1.53-1.39(\mathrm{~m}, 6 \mathrm{H}), 1.38-$ $1.22(\mathrm{~m}, 5 \mathrm{H}), 1.17-0.98(\mathrm{~m}, 3 \mathrm{H}), 0.96-0.91(\mathrm{~m}, 6 \mathrm{H}), 0.67(\mathrm{~s}, 3 \mathrm{H}), 2 \times \mathrm{H}$ not observed (exchangeable). ${ }^{13} \mathrm{C} \mathrm{NMR}\left(\mathrm{CDCl}_{3}, 101 \mathrm{MHz}\right): \delta 172.4,154.8,139.9,135.3,128.5,126.4$, $71.54,71.47,66.0,55.9,55.1,45.4,43.9,42.6,40.3,39.3,37.4,37.0,35.6,35.1,34.2,31.5$, $30.5,28.8,27.0,23.5,21.3,18.8,12.3 .2 \times C$ not observed. HRMS: exact mass calculated for $[\mathrm{M}+\mathrm{Na}]^{+}\left(\mathrm{C}_{36} \mathrm{H}_{52} \mathrm{Cl}_{2} \mathrm{~N}_{2} \mathrm{NaO}_{5}\right)$ requires $685.3145 \mathrm{~m} / \mathrm{z}$, found $685.3119 \mathrm{~m} / \mathrm{z}$. 
yl)pentanamido)ethyl)carbamate (5). Prepared according to General Procedure D with ursodeoxycholic acid (176 mg, $0.45 \mathrm{mmol}, 1.1$ equiv), $N, N$-diisopropylethylamine (286 $\mu \mathrm{L}$, 1.64 mmol, 4.0 equiv), HATU (171 mg, 0.45 mmol, 1.1 equiv), 3,5-dichlorobenzyl (2aminoethyl)carbamate (107 mg, $0.41 \mathrm{mmol}, 1.0$ equiv) and DMF (2 mL). The crude material was subjected to the purification procedure outlined in the General Procedure (silica gel, 0$10 \% \mathrm{MeOH}$ in $\mathrm{CH}_{2} \mathrm{Cl}_{2}$ ) to give the desired product as a clear solid (134 mg, $51 \%$ ). Umax (neat): 3329, 2933, 2864, 1706, 1652, $1574 \mathrm{~cm}^{-1} .{ }^{1} \mathrm{H} \mathrm{NMR}\left(\mathrm{CDCl}_{3}, 400 \mathrm{MHz}\right): \delta$ 7.30-7.27 (m, 1H), 7.23-7.19 (m, 2H), 6.34 (br. s, 1H), 5.73 (br. s, 1H), 5.01 (s, 2H), 3.65-3.50 (m, 2H), 3.42-3.25 (m, 4H), 2.27-2.12 (m, 1H), 2.11-2.00 (m, 1H), 2.00-1.93 (m, 1H), 1.88-1.71 (m, $5 \mathrm{H}), 1.70-1.61(\mathrm{~m}, 2 \mathrm{H}), 1.60-1.53(\mathrm{~m}, 2 \mathrm{H}), 1.50-1.35(\mathrm{~m}, 5 \mathrm{H}), 1.35-1.16(\mathrm{~m}, 6 \mathrm{H}), 1.14-0.98$ $(\mathrm{m}, 3 \mathrm{H}), 0.97-0.87(\mathrm{~m}, 6 \mathrm{H}), 0.64(\mathrm{~s}, 3 \mathrm{H}), 2 \times \mathrm{H}$ not observed (exchangeable). ${ }^{13} \mathrm{C}$ NMR $\left(\mathrm{CDCl}_{3}, 101 \mathrm{MHz}\right): \delta 175.0,156.9,140.1,135.2,128.2,126.1,71.4,65.1,56.01,55.95,55.1$ $43.8,42.6,41.2,40.3,39.9,39.4,37.5,37.2,35.6,35.1,34.2,33.6,32.0,30.4,28.8,27.0$, 23.5, 21.3, 18.6, 12.2. $1 \times \mathrm{C}$ not observed. HRMS: exact mass calculated for $[\mathrm{M}+\mathrm{Na}]^{+}$ $\left(\mathrm{C}_{34} \mathrm{H}_{50} \mathrm{Cl}_{2} \mathrm{~N}_{2} \mathrm{NaO}_{5}\right)$ requires $659.2989 \mathrm{~m} / \mathrm{z}$, found $659.2965 \mathrm{~m} / \mathrm{z}$.

\section{3,5-Dichlorobenzyl}

(3-((4R)-4-((3R,7S,10S,13R)-3,7-dihydroxy-10,13dimethylhexadecahydro-1H-cyclopenta[a]phenanthren-17-

yl)pentanamido)propyl)carbamate (6). Prepared according to General Procedure D with ursodeoxycholic acid (156 mg, $0.39 \mathrm{mmol}, 1.1$ equiv), $N, N$-diisopropylethylamine (314 $\mu \mathrm{L}$, 1.8 mmol, 4.0 equiv), HATU (151 mg, $0.39 \mathrm{mmol}, 1.1$ equiv), 3,5-dichlorobenzyl (3aminopropyl)carbamate (100 mg, $0.36 \mathrm{mmol}, 1.0$ equiv) and DMF (1 mL). The crude material was subjected to the purification outlined in the General Procedure (silica gel, 0$10 \% \mathrm{MeOH}$ in $\mathrm{CH}_{2} \mathrm{Cl}_{2}$ ) to give the desired product as a clear solid (39 $\mathrm{mg}, 16 \%$ ). Umax (neat): $3813,2933,2866,1708,1654,1574,1542 \mathrm{~cm}^{-1} .{ }^{1} \mathrm{H}$ NMR $\left(\mathrm{CDCl}_{3}, 400 \mathrm{MHz}\right): \delta 7.31-$ $7.28(\mathrm{~m}, 1 \mathrm{H}), 7.25-7.21(\mathrm{~m}, 2 \mathrm{H}), 5.92(\mathrm{~s}, 1 \mathrm{H}), 5.33(\mathrm{~s}, 1 \mathrm{H}), 5.03(\mathrm{~s}, 2 \mathrm{H}), 3.62-3.55(\mathrm{~m}, 2 \mathrm{H})$, 3.51-3.46 (m, 2H), 3.35-3.31 (m, 2H), 3.26-3.18 (m, 2H), 2.29-2.20 (m, 1H), 2.16-2.04 (m, 
1H), 2.03-1.96 (m, 1H), 1.95-1.86 (m, 1H), 1.84-1.74 (m, 4H), 1.71-1.55 (m, 7H), 1.53-1.39 $(\mathrm{m}, 6 \mathrm{H}), 1.37-1.21(\mathrm{~m}, 4 \mathrm{H}), 1.14(\mathrm{td}, J=3.1,12.6 \mathrm{~Hz}, 1 \mathrm{H}), 1.10-0.98(\mathrm{~m}, 2 \mathrm{H}), 0.97-0.90(\mathrm{~m}$, $6 \mathrm{H}), 0.67(\mathrm{~s}, 3 \mathrm{H}) .{ }^{13} \mathrm{C} \mathrm{NMR}\left(\mathrm{CDCl}_{3}, 101 \mathrm{MHz}\right): \delta 174.4,156.6,140.3,135.2,128.2,126.1$, $71.6,71.5,65.1,55.9,55.1,43.9,42.6,40.3,39.3,37.7,37.5,37.0,36.0,35.6,35.1,34.2$, 33.8, 32.0, 30.5, 30.2, 28.8, 27.0, 23.5, 21.3, 18.6, 12.3. 1 × C not observed. HRMS: exact mass calculated for $[\mathrm{M}+\mathrm{Na}]^{+}\left(\mathrm{C}_{35} \mathrm{H}_{52} \mathrm{Cl}_{2} \mathrm{~N}_{2} \mathrm{NaO}_{5}\right)$ requires $673.3145 \mathrm{~m} / \mathrm{z}$, found $673.3113 \mathrm{~m} / \mathrm{z}$.

(4R)-N-(3,5-Dichlorobenzyl)-4-((3R,7S,10S,13R)-3,7-dihydroxy-10,13-

dimethylhexadecahydro-1H-cyclopenta[a]phenanthren-17-yl)pentanamide

Prepared according to General Procedure D with ursodeoxycholic acid (100 mg, $0.26 \mathrm{mmol}$, 1.0 equiv), $N, N$-diisopropylethylamine (180 $\mu \mathrm{L}, 1.02 \mathrm{mmol}, 4.0$ equiv), HATU (97 mg, 0.26 mmol, 1.0 equiv), 3,5-dichlorobenzylamine (35 $\mu \mathrm{L}, 0.26 \mathrm{mmol}, 1.0$ equiv) and DMF ( $1 \mathrm{~mL})$. The crude material was subjected to the purification outlined in the General Procedure (silica gel, $0-10 \% \mathrm{MeOH}$ in $\mathrm{CH}_{2} \mathrm{Cl}_{2}$ ) to afford the desired product as a white solid (14 $\mathrm{mg}, 10 \%$ ). $U_{\max }$ (neat): 3296, 2931, 2866, 1654, 1574, $1434 \mathrm{~cm}^{-1} .{ }^{1} \mathrm{H} \mathrm{NMR}\left(\mathrm{CDCl}_{3}, 400 \mathrm{MHz}\right): \delta 7.28-$ $7.25(\mathrm{~m}, 1 \mathrm{H}), 7.15(\mathrm{~d}, J=1.9 \mathrm{~Hz}, 2 \mathrm{H}), 5.80(\mathrm{~s}, 1 \mathrm{H}), 4.39(\mathrm{~d}, J=6.1 \mathrm{~Hz}, 2 \mathrm{H}), 3.64-3.54(\mathrm{~m}$, 2H), 2.35-2.25 (m, 1H), 2.18-2.09 (m, 1H), $1.99(\mathrm{dt}, J=3.0,12.4 \mathrm{~Hz}, 1 \mathrm{H}), 1.94-1.85(\mathrm{~m}, 1 \mathrm{H})$, 1.85-1.74 (m, 3H), 1.70-1.63 (m, 2H), 1.62-1.55 (m, 3H), 1.53-1.39 (m, 6H), 1.38-1.20 (m, $6 \mathrm{H}), 1.17-0.97(\mathrm{~m}, 3 \mathrm{H}), 0.96-0.91(\mathrm{~m}, 6 \mathrm{H}), 0.67(\mathrm{~s}, 3 \mathrm{H}) .{ }^{13} \mathrm{C} \mathrm{NMR}\left(\mathrm{CDCl}_{3}, 101 \mathrm{MHz}\right): \delta 173.6$, $142.1,135.4,127.8,126.2,71.6,71.5,55.9,55.1,43.9,42.7,42.6,40.3,39.3,37.5,37.0$, $35.5,35.1,34.2,33.7,31.9,30.5,28.8,27.0,23.5,21.3,18.6,12.3 .1 \times C$ not observed. HRMS: exact mass calculated for $[\mathrm{M}+\mathrm{Na}]^{+}\left(\mathrm{C}_{31} \mathrm{H}_{45} \mathrm{Cl}_{2} \mathrm{NNaO}_{3}\right)$ requires $572.2669 \mathrm{~m} / \mathrm{z}$, found $572.2650 \mathrm{~m} / \mathrm{z}$.

(4R)-N-(2-Aminoethyl)-4-((3R,7S,10S,13R)-3,7-dihydroxy-10,13-

dimethylhexadecahydro-1H-cyclopenta[a]phenanthren-17-yl)pentanamide

(8).

Prepared according to General Procedure C, using tert-butyl (2-((4R)-4-((3R,7S,10S,13R)- 
3,7-dihydroxy-10,13-dimethylhexadecahydro-1H-cyclopenta[a]phenanthren-17-

yl)pentanamido)ethyl)carbamate (936 mg, $1.75 \mathrm{mmol}, 1.0$ equiv), trifluoroacetic acid (2 mL), $\mathrm{CH}_{2} \mathrm{Cl}_{2}(4 \mathrm{~mL})$, diluted with $1 \mathrm{M} \mathrm{NaOH}(100 \mathrm{~mL})$, the organics were extracted with ethyl acetate $(100 \mathrm{~mL})$, washed with brine $(100 \mathrm{~mL})$, dried with a hydrophobic frit and concentrated in vacuo to afford the desired product as a white solid (605 mg, $63 \%$ ). Umax (neat): 3283, 2935, 2669, 1715, 1654, $1157 \mathrm{~cm}^{-1} .{ }^{1} \mathrm{H}$ NMR $\left(\mathrm{CDCl}_{3}, 500 \mathrm{MHz}\right): \delta 9.36(\mathrm{~s}, 1 \mathrm{H})$, $7.89(\mathrm{~s}, 1 \mathrm{H}), 4.42(\mathrm{~d}, J=4.6 \mathrm{~Hz}, 1 \mathrm{H}), 3.85(\mathrm{~d}, J=6.8 \mathrm{~Hz}, 1 \mathrm{H}), 3.21(\mathrm{t}, J=5.4 \mathrm{~Hz}, 2 \mathrm{H}), 3.17$ $(\mathrm{t}, J=5.5 \mathrm{~Hz}, 2 \mathrm{H}), 2.13-2.03(\mathrm{~m}, 1 \mathrm{H}), 1.99-1.90(\mathrm{~m}, 2 \mathrm{H}), 1.86-1.80(\mathrm{~m}, 1 \mathrm{H}), 1.86-1.80(\mathrm{~m}$, 1H), $1.80-1.71(m, 1 H), 1.70-1.60(m, 3 H), 1.51-1.43(m, 3 H), 1.42-1.26(m, 7 H), 1.23-1.06$ $(\mathrm{m}, 3 \mathrm{H}), 1.03-0.92(\mathrm{~m}, 2 \mathrm{H}), 0.89-0.85(\mathrm{~m}, 6 \mathrm{H}), 0.61(\mathrm{~s}, 3 \mathrm{H}) \cdot{ }^{13} \mathrm{C} \mathrm{NMR}\left(\mathrm{CDCl}_{3}, 101 \mathrm{MHz}\right): \delta$ $173.0,69.7,69.4,55.9,54.7,43.1,43.0,42.2,39.8,39.2,38.7,37.7,37.30,37.26,34.9$, 34.8, 33.7, 32.4, 31.5, 30.2, 28.1, 26.7, 23.3, 20.8, 18.4, 12.0. HRMS: exact mass calculated for $[\mathrm{M}+\mathrm{H}]^{+}\left(\mathrm{C}_{26} \mathrm{H}_{47} \mathrm{~N}_{2} \mathrm{O}_{3}\right)$ requires $435.3581 \mathrm{~m} / \mathrm{z}$, found $435.3576 \mathrm{~m} / \mathrm{z}$.

\section{Benzyl (2-((4R)-4-((3R,7S,10S,13R)-3,7-dihydroxy-10,13-dimethylhexadecahydro-1H-} cyclopenta[a]phenanthren-17-yl)pentanamido)ethyl)carbamate (9). Prepared according to General Procedure D with ursodeoxycholic acid (445 mg, $1.13 \mathrm{mmol}, 1.1$ equiv), N,Ndiisopropylethylamine (720 $\mu \mathrm{L}, 4.12 \mathrm{mmol}, 4.0$ equiv), HATU (430 mg, $0.13 \mathrm{mmol}, 1.1$ equiv), benzyl (2-aminoethyl)carbamate (200 mg, $1.03 \mathrm{mmol}, 1.0$ equiv) and DMF (5 mL). The crude material was subjected to the purification outlined in the General Procedure (silica gel, $0-10 \% \mathrm{MeOH}$ in $\mathrm{CH}_{2} \mathrm{Cl}_{2}$ ) to afford the desired product as a clear solid (399 $\mathrm{mg}, 68 \%$ ). umax (neat): $3298,2933,2866,1706,1652,1527 \mathrm{~cm}^{-1} .{ }^{1} \mathrm{H}$ NMR $\left(\mathrm{CDCl}_{3}, 500 \mathrm{MHz}\right): \delta$ 7.39-7.28 (m, $5 \mathrm{H}), 6.10(\mathrm{~s}, 1 \mathrm{H}), 5.29(\mathrm{~s}, 1 \mathrm{H}), 5.09(\mathrm{~s}, 2 \mathrm{H}), 3.63-3.52(\mathrm{~m}, 2 \mathrm{H}), 3.41-3.28(\mathrm{~m}, 4 \mathrm{H}), 2.21-2.16$ $(\mathrm{m}, 1 \mathrm{H}), 2.08-2.01(\mathrm{~m}, 1 \mathrm{H}), 2.01-1.96(\mathrm{~m}, 1 \mathrm{H}), 1.91-1.84(\mathrm{~m}, 1 \mathrm{H}), 1.82-1.75(\mathrm{~m}, 3 \mathrm{H}), 1.73-$ $1.64(\mathrm{~m}, 4 \mathrm{H}), 1.61-1.54(\mathrm{~m}, 3 \mathrm{H}), 1.52-1.39(\mathrm{~m}, 5 \mathrm{H}), 1.34-1.20(\mathrm{~m}, 6 \mathrm{H}), 1.16-1.09(\mathrm{~m}, 1 \mathrm{H})$, 1.06-0.99 (m, 2H), 0.95-0.88 (m, 6H), $\left.0.67(\mathrm{~s}, 3 \mathrm{H}) .{ }^{13} \mathrm{C} \mathrm{NMR} \mathrm{(101} \mathrm{MHz,} \mathrm{CDCl}_{3}\right): \delta$ 174.6, $157.4,136.5,128.7,128.3,128.2,71.6,71.5,67.0,55.9,55.1,43.9,42.6,41.2,40.3,39.4$, 
$37.5,37.0,35.6,35.1,34.2,33.7,31.9,30.5,28.8,27.0,23.5,21.3,18.6,12.3 .2 \times C$ not observed. HRMS: exact mass calculated for $[\mathrm{M}+\mathrm{Na}]^{+}\left(\mathrm{C}_{34} \mathrm{H}_{52} \mathrm{~N}_{2} \mathrm{NaO}_{5}\right)$ requires $591.3768 \mathrm{~m} / \mathrm{z}$, found $591.3746 \mathrm{~m} / \mathrm{z}$.

\section{Benzyl-4-((4R)-4-((3R,7S,10S,13R)-3,7-dihydroxy-10,13-dimethylhexadecahydro-1H-} cyclopenta[a]phenanthren-17-yl)pentanoyl)piperazine-1-carboxylate (10). Prepared according to General Procedure D with ursodeoxycholic acid (500 mg, $1.27 \mathrm{mmol}, 1.1$ equiv), $\mathrm{N}, \mathrm{N}$-diisopropylethylamine $(450 \mu \mathrm{L}, 2.57 \mathrm{mmol}, 2.0$ equiv), HATU (243 mg, $1.27 \mathrm{mmol}, 1.1$ equiv), benzylpiperazine-1-carboxylate $(223 \mu \mathrm{L}, 1.16 \mathrm{mmol}, 1.0$ equiv) and DMF $(5 \mathrm{~mL})$. The crude material was subjected to the purification outlined in the General Procedure (silica gel, $0-10 \% \mathrm{MeOH}$ in $\mathrm{CH}_{2} \mathrm{Cl}_{2}$ ) to afford the desired product as a clear solid (258 $\mathrm{mg}, 37 \%$ ). $U_{\max }$ (neat): 3374, 2929, 2866, 1702, $1630 \mathrm{~cm}^{-1} .{ }^{1} \mathrm{H}$ NMR $\left(\mathrm{CDCl}_{3}, 500 \mathrm{MHz}\right): \delta$ 7.39-7.30 (m, $5 \mathrm{H}), 5.15(\mathrm{~s}, 2 \mathrm{H}), 3.63-3.54(\mathrm{~m}, 4 \mathrm{H}), 3.56-3.40(\mathrm{~m}, 7 \mathrm{H}), 2.41-2.33(\mathrm{~m}, 1 \mathrm{H}), 2.26-2.17(\mathrm{~m}, 1 \mathrm{H})$, $1.99(\mathrm{~d}, J=12.5 \mathrm{~Hz}, 1 \mathrm{H}), 1.95-1.85(\mathrm{~m}, 1 \mathrm{H}), 1.84-1.73(\mathrm{~m}, 4 \mathrm{H}), 1.71-1.63(\mathrm{~m}, 3 \mathrm{H}), 1.62-1.55$ (m, 2H), 1.52-1.39 (m, 6H), 1.39-1.21 (m, 5H), 1.19-0.97 (m, 3H), 0.97-0.91 (m, 6H), 0.67 (s, 3H). ${ }^{13} \mathrm{C} \mathrm{NMR}\left(\mathrm{CDCl}_{3}, 101 \mathrm{MHz}\right): \delta 172.4,155.3,136.5,128.7,128.4,128.2,71.6,71.5$, $67.6,55.9,55.1,45.5,43.9,42.6,41.4,40.3,39.3,37.4,37.0,35.6,35.1,34.2,31.5,30.5$, 28.8, 27.0, 23.5, 21.3, 18.8, 12.3. $2 \times$ aliphatic $C$ not observed. HRMS: exact mass calculated for $[\mathrm{M}+\mathrm{Na}]^{+}\left(\mathrm{C}_{36} \mathrm{H}_{54} \mathrm{~N}_{2} \mathrm{NaO}_{5}\right)$ requires $617.3925 \mathrm{~m} / \mathrm{z}$, found $617.3915 \mathrm{~m} / \mathrm{z}$.

\section{3,5-Dichlorobenzyl-4-((4R)-4-((3R,7S,10S,13R)-3,7-dihydroxy-10,13-} dimethylhexadecahydro-1H-cyclopenta[a]phenanthren-17-yl)pentyl)piperazine-1carboxylate (11). To a round bottom flask at $0{ }^{\circ} \mathrm{C}$ was added 3,5-dichlorobenzyl 4-((4R)-4$((3 R, 7 S, 10 S, 13 R)-3,7-d i h y d r o x y-10,13-d i m e t h y l h e x a d e c a h y d r o-1 H-$

cyclopenta[a]phenanthren-17-yl)pentanoyl)piperazine-1-carboxylate (92 mg, $0.14 \mathrm{mmol}, 1.0$ equiv), THF ( $5 \mathrm{~mL}), \mathrm{BH}_{3}$.THF ( $1 \mathrm{M}, 700 \mu \mathrm{L}, 0.70 \mathrm{mmol}, 5.0$ equiv) and the reaction stirred at $70{ }^{\circ} \mathrm{C}$ for $16 \mathrm{~h}$. The reaction mixture was cooled to room temperature, quenched with $1 \mathrm{M}$ 
$\mathrm{HCl}(20 \mathrm{~mL})$ and stirred for $1 \mathrm{~h}$. The organics were extracted with ethyl acetate $(20 \mathrm{~mL})$, washed with brine $(20 \mathrm{~mL})$, dried with a hydrophobic frit and concentrated in vacuo. The crude material was purified by column chromatography on silica $\left(0-100 \% \mathrm{MeOH}\right.$ in $\left.\mathrm{CH}_{2} \mathrm{Cl}_{2}\right)$ to afford the desired product as a white solid (4 mg, 4\%). $u_{\max }$ (neat): 3381, 2929, 2866, 1708, $1635 \mathrm{~cm}^{-1} .{ }^{1} \mathrm{H} \mathrm{NMR}\left(\mathrm{CDCl}_{3}, 500 \mathrm{MHz}\right): \delta 7.33-7.31(\mathrm{~m}, 1 \mathrm{H}), 7.23(\mathrm{~d}, J=1.7 \mathrm{~Hz}, 2 \mathrm{H})$, $5.08(\mathrm{~s}, 2 \mathrm{H}), 3.66-3.43(\mathrm{~m}, 12 \mathrm{H}), 2.43-2.34(\mathrm{~m}, 1 \mathrm{H}), 2.29-2.18(\mathrm{~m}, 1 \mathrm{H}), 2.03-1.97(\mathrm{~m}, 1 \mathrm{H})$, 1.96-1.86 (m, 1H), 1.85-1.74 (m, 4H), 1.71-1.62 (m, 2H), 1.62-1.53 (m, 4H), 1.52-1.41 (m, $6 \mathrm{H}), 1.39-1.32(\mathrm{~m}, 2 \mathrm{H}), 1.29-1.21(\mathrm{~m}, 2 \mathrm{H}), 1.19-1.11(\mathrm{~m}, 2 \mathrm{H}), 1.11-1.01(\mathrm{~m}, 2 \mathrm{H}), 0.99-0.92$ (m, 6H), $0.68(\mathrm{~s}, 3 \mathrm{H}) .{ }^{13} \mathrm{C} \mathrm{NMR}\left(\mathrm{CDCl}_{3}, 126 \mathrm{MHz}\right): \delta 172.4,139.9,135.3,128.5,126.4,71.6$, $71.5,66.0,55.9,55.1,51.0,45.5,43.9,42.6,40.3,39.3,37.5,37.0,35.7,35.1,34.2,31.5$, $30.5,28.9,27.1,23.5,21.3,18.8,12.3 .3 \times C$ not observed. HRMS: exact mass calculated for $[\mathrm{M}+\mathrm{H}]^{+}\left(\mathrm{C}_{36} \mathrm{H}_{55} \mathrm{Cl}_{2} \mathrm{~N}_{2} \mathrm{O}_{4}\right)$ requires $649.3533 \mathrm{~m} / \mathrm{z}$, found $649.3506 \mathrm{~m} / \mathrm{z}$.

\section{3,5-Dichlorobenzyl-4-(((4R)-4-((3R,7S,10S,13R)-3,7-dihydroxy-10,13-}

\section{dimethylhexadecahydro-1H-cyclopenta[a]phenanthren-17-}

yl)pentanamido)methyl)piperidine-1-carboxylate (12). Prepared according to General Procedure D with ursodeoxycholic acid (136 mg, $0.35 \mathrm{mmol}, 1.1$ equiv), N,Ndiisopropylethylamine (223 $\mu \mathrm{L}, 1.28 \mathrm{mmol}, 4.0$ equiv), HATU (133 mg, $0.35 \mathrm{mmol}, 1.1$ equiv), 3,5-dichlorobenzyl 4-(aminomethyl)piperidine-1-carboxylate (100 mg, $0.32 \mathrm{mmol}, 1.0$ equiv) and DMF (2 $\mathrm{mL})$. The crude material was subjected to the purification outlined in the General Procedure (silica gel, $0-10 \% \mathrm{MeOH}$ in $\mathrm{CH}_{2} \mathrm{Cl}_{2}$ ) to afford the desired product as a clear solid (80 mg, 36 \%). Umax (neat): 3323, 2931, 2866, 1691, $1654 \mathrm{~cm}^{-1} .{ }^{1} \mathrm{H} \mathrm{NMR}\left(\mathrm{CDCl}_{3}\right.$, $400 \mathrm{MHz}): \delta$ 7.31-7.29 (m, 1H), 7.23-7.20 (m, 2H), $5.52(\mathrm{~s}, 1 \mathrm{H}), 5.05(\mathrm{~s}, 2 \mathrm{H}), 4.27-4.09(\mathrm{~m}$, $2 \mathrm{H}), 3.65-3.53(\mathrm{~m}, 2 \mathrm{H}), 3.26-3.08(\mathrm{~m}, 2 \mathrm{H}), 2.89-2.79(\mathrm{~m}, 2 \mathrm{H}), 2.30-2.21(\mathrm{~m}, 1 \mathrm{H}), 2.14-2.03$ $(m, 1 H), 2.02-1.96(m, 1 H), 1.95-1.86(m, 1 H), 1.85-1.75(m, 3 H), 1.74-1.63(m, 4 H), 1.63-$ $1.55(\mathrm{~m}, 4 \mathrm{H}), 1.55-1.39(\mathrm{~m}, 6 \mathrm{H}), 1.38-1.12(\mathrm{~m}, 7 \mathrm{H}), 1.10-0.98(\mathrm{~m}, 2 \mathrm{H}), 0.97-0.90(\mathrm{~m}, 6 \mathrm{H})$, $0.67(\mathrm{~s}, 3 \mathrm{H}) .{ }^{13} \mathrm{C} \mathrm{NMR}\left(\mathrm{CDCl}_{3}, 101 \mathrm{MHz}\right): \delta 173.8,154.8,140.4,135.2,128.2,126.2,71.6$, 
$71.5,65.6,55.9,55.1,44.9,44.0,43.9,42.6,40.3,39.3,37.5,37.0,36.3,35.5,35.1,34.2$, 33.8, 32.0, 30.5, 29.8, 28.8, 27.0, 23.5, 21.3, 28.6, 12.3. $1 \times \mathrm{C}$ not observed. HRMS: exact mass calculated for $[\mathrm{M}+\mathrm{Na}]^{+}\left(\mathrm{C}_{38} \mathrm{H}_{56} \mathrm{Cl}_{2} \mathrm{~N}_{2} \mathrm{NaO}_{5}\right)$ requires $713.3458 \mathrm{~m} / \mathrm{z}$, found $713.3425 \mathrm{~m} / \mathrm{z}$.

\section{3,5-Dichlorobenzyl}

((1-((4R)-4-((3R,7S,10S,13R)-3,7-dihydroxy-10,13-

\section{dimethylhexadecahydro-1H-cyclopenta[a]phenanthren-17-yl)pentanoyl)-4-}

hydroxypiperidin-4-yl)methyl)carbamate (13). Prepared according to General Procedure

D with ursodeoxycholic acid (67 mg, $0.17 \mathrm{mmol}, 1.1$ equiv), $N, N$-diisopropylethylamine (131 $\mu \mathrm{L}, 0.75 \mathrm{mmol}, 5.0$ equiv), HATU (65 mg, $0.17 \mathrm{mmol}, 1.1$ equiv), 3,5-dichlorobenzyl ((4hydroxypiperidin-4-yl)methyl)carbamate (50 mg, $0.15 \mathrm{mmol}, 1.0$ equiv) and DMF (1 mL). The crude material was subjected to the purification outlined in the General Procedure (silica gel, $0-10 \% \mathrm{MeOH}$ in $\mathrm{CH}_{2} \mathrm{Cl}_{2}$ ) to afford the desired product as a clear solid (5.1 mg, $3 \%$ ). $u_{\max }$ (neat): 3357, 2929, 2866, 1702, 1618, $1574 \mathrm{~cm}^{-1} .{ }^{1} \mathrm{H} \mathrm{NMR}\left(\mathrm{CDCl}_{3}, 400 \mathrm{MHz}\right): \delta$ 7.34-7.28 (m, 1H), $7.25(\mathrm{~d}, J=1.7 \mathrm{~Hz}, 2 \mathrm{H}), 5.28(\mathrm{~s}, 1 \mathrm{H}), 5.05(\mathrm{~s}, 2 \mathrm{H}), 4.26(\mathrm{~d}, J=12.9 \mathrm{~Hz}, 1 \mathrm{H}), 3.64-3.54$ (m, 3H), $3.43(\mathrm{t}, J=11.4 \mathrm{~Hz}, 1 \mathrm{H}), 3.34-3.25(\mathrm{~m}, 1 \mathrm{H}), 3.22-3.15(\mathrm{~m}, 1 \mathrm{H}), 3.11(\mathrm{t}, J=11.4 \mathrm{~Hz}$, 1H), 2.45-2.34 (m, 1H), 2.32-2.19 (m, 1H), 2.04-1.97 (m, 1H), 1.96-1.86 (m, 1H), 1.85-1.73 $(\mathrm{m}, 4 \mathrm{H}), 1.69-1.64(\mathrm{~m}, 2 \mathrm{H}), 1.62-1.54(\mathrm{~m}, 6 \mathrm{H}), 1.53-1.47(\mathrm{~m}, 3 \mathrm{H}), 1.46-1.40(\mathrm{~m}, 4 \mathrm{H}), 1.38-$ $1.28(\mathrm{~m}, 5 \mathrm{H}), 1.19-0.98(\mathrm{~m}, 3 \mathrm{H}), 0.97-0.90(\mathrm{~m}, 6 \mathrm{H}), 0.70(\mathrm{~s}, 3 \mathrm{H}) .{ }^{13} \mathrm{C}$ NMR $\left(\mathrm{CDCl}_{3}, 101\right.$ $\mathrm{MHz}): \delta 172.2,157.3,139.8,135.3,128.4,126.2,71.6,71.5,70.4,65.6,55.9,55.1,51.4$ $43.9,42.6,41.7,40.3,39.3,37.5,37.4,37.0,35.7,35.5,35.1,34.8,34.2,31.7,30.52,30.48$, 28.8, 27.1, 23.5, 21.3, 18.8, 12.3. Rotameric mixture observed. HRMS: exact mass calculated for $[\mathrm{M}+\mathrm{Na}]^{+}\left(\mathrm{C}_{38} \mathrm{H}_{56} \mathrm{Cl}_{2} \mathrm{~N}_{2} \mathrm{NaO}_{6}\right)$ requires $729.3408 \mathrm{~m} / \mathrm{z}$, found $729.3372 \mathrm{~m} / \mathrm{z}$.

\section{2-(3,5-Dichlorobenzyl)-7-((4R)-4-((3R,7S,10S,13R)-3,7-dihydroxy-10,13-}

dimethylhexadecahydro-1H-cyclopenta[a]phenanthren-17-

yl)pentanoyl)hexahydroimidazo[1,5-a]pyrazin-3(2H)-one (14). Prepared according to General Procedure D with ursodeoxycholic acid $(120 \mathrm{mg}, 0.31 \mathrm{mmol}, 1.1$ equiv), N,N- 
diisopropylethylamine (244 $\mu \mathrm{L}, 1.50 \mathrm{mmol}, 5.0$ equiv), HATU (117 mg, $0.31 \mathrm{mmol}, 1.1$ equiv), 2-(3,5-dichlorobenzyl)hexahydroimidazo[1,5-a]pyrazin-3(2H)-one (73 mg, $0.32 \mathrm{mmol}, 1.0$ equiv) and DMF (2 mL). The crude material was subjected to the purification outlined in the General Procedure (silica gel, $0-10 \% \mathrm{MeOH}$ in $\mathrm{CH}_{2} \mathrm{Cl}_{2}$ ) to afford the desired product as a clear solid as an epimeric mixture (80 mg, 36\%). Umax (neat): 3404, 2931, 2864, 1691, 1635 , $1429 \mathrm{~cm}^{-1} .{ }^{1} \mathrm{H}$ NMR $\left(\mathrm{CDCl}_{3}, 400 \mathrm{MHz}, 323 \mathrm{~K}\right): \delta$ 7.30-7.27 (m, 1H), 7.16-7.12 (m, 2H), 4.72$4.57(\mathrm{~m}, 1 \mathrm{H}), 4.38(\mathrm{~d}, J=15.4 \mathrm{~Hz}, 1 \mathrm{H}), 4.29(\mathrm{~d}, J=15.5 \mathrm{~Hz}, 1 \mathrm{H}), 3.95(\mathrm{~d}, J=12.1 \mathrm{~Hz}, 1 \mathrm{H})$, 3.84-3.71 (m, 1H), 3.64-3.53 (m, 3H), $3.35(\mathrm{t}, J=8.6 \mathrm{~Hz}, 1 \mathrm{H}), 3.20-3.04(\mathrm{~m}, 1 \mathrm{H}), 3.01-2.84$ (m, 2H), 2.43-2.32 (m, 1H), 2.32-2.19 (m, 1H), $2.00(\mathrm{~d}, J=12.5 \mathrm{~Hz}, 1 \mathrm{H}), 1.95-1.75(\mathrm{~m}, 5 \mathrm{H})$, $1.71-1.65(\mathrm{~m}, 2 \mathrm{H}), \quad 1.63-1.56(\mathrm{~m}, 2 \mathrm{H}), 1.56-1.40(\mathrm{~m}, 8 \mathrm{H}), 1.38-1.02(\mathrm{~m}, 8 \mathrm{H}), 1.00-0.92(\mathrm{~m}$, $6 \mathrm{H}), 0.67(\mathrm{~s}, 3 \mathrm{H}) .{ }^{13} \mathrm{C} \mathrm{NMR}\left(\mathrm{CDCl}_{3}, 101 \mathrm{MHz}\right): \delta 172.6,159.0,140.5,135.5,128.0,126.5$, $71.51,71.46,60.5,55.8,55.1,50.8,47.3,46.0,45.5,45.3,43.9,42.5,41.2,40.3,39.3,37.4$, $37.0,35.6,35.0,34.2,31.5,30.5,30.4,28.8,27.0,23.5,21.3,18.8,12.3$. HRMS: exact mass calculated for $[\mathrm{M}+\mathrm{Na}]^{+}\left(\mathrm{C}_{37} \mathrm{H}_{53} \mathrm{Cl}_{2} \mathrm{~N}_{3} \mathrm{NaO}_{4}\right)$ requires $696.3305 \mathrm{~m} / \mathrm{z}$, found $696.3289 \mathrm{~m} / \mathrm{z}$.

\section{3,5-Dichlorobenzyl-3-((4R)-4-((3R,7S,10S,13R)-3,7-dihydroxy-10,13-}

dimethylhexadecahydro-1H-cyclopenta[a]phenanthren-17-yl)pentanamido)azetidine-1carboxylate (15). Prepared according to General Procedure D with ursodeoxycholic acid (157 mg, 0.40 mmol, 1.1 equiv), $N, N$-diisopropylethylamine (314 $\mu \mathrm{L}, 1.8 \mathrm{mmol}, 4.0$ equiv), HATU (152 mg, 0.40 mmol, 1.1 equiv), 3,5-dichlorobenzyl 3-aminoazetidine-1-carboxylate (100 mg, $0.36 \mathrm{mmol}, 1.0$ equiv) and DMF (1 mL). The crude material was subjected to the

purification outlined in the General Procedure (silica gel, 0-10\% $\mathrm{MeOH}$ in $\mathrm{CH}_{2} \mathrm{Cl}_{2}$ ) to afford the desired product as a clear solid (108 mg, $46 \%$ ). Umax (neat): 3400, 2933, 2865, 1699, 1654, 1574, $1419 \mathrm{~cm}^{-1} .{ }^{1} \mathrm{H}$ NMR $\left(\mathrm{CDCl}_{3}, 500 \mathrm{MHz}\right): \delta$ 7.30-7.27 (m, 1H), 7.21-7.17 (m, 2H), 6.51 (br. s, 1H), $5.00(\mathrm{~s}, 2 \mathrm{H}), 4.69-4.62(\mathrm{~m}, 1 \mathrm{H}), 4.35-4.28(\mathrm{~m}, 2 \mathrm{H}), 3.83(\mathrm{dd}, J=5.2,9.3 \mathrm{~Hz}$, $2 \mathrm{H}), 3.60-3.51(\mathrm{~m}, 2 \mathrm{H}), 2.31-2.19(\mathrm{~m}, 1 \mathrm{H}), 2.12-2.02(\mathrm{~m}, 1 \mathrm{H}), 2.02-1.94(\mathrm{~m}, 1 \mathrm{H}), 1.93-1.84$ $(\mathrm{m}, 3 \mathrm{H}), 1.82-1.72(\mathrm{~m}, 4 \mathrm{H}), 1.69-1.62(\mathrm{~m}, 2 \mathrm{H}), 1.61-1.54(\mathrm{~m}, 2 \mathrm{H}), 1.50-1.45(\mathrm{~m}, 2 \mathrm{H}), 1.44-$ 
$1.39(\mathrm{~m}, 4 \mathrm{H}), 1.35-1.18(\mathrm{~m}, 5 \mathrm{H}), 1.16-0.98(\mathrm{~m}, 3 \mathrm{H}), 0.95-0.88(\mathrm{~m}, 6 \mathrm{H}), 0.65(\mathrm{~s}, 3 \mathrm{H}) .{ }^{13} \mathrm{C}$ NMR $\left(\mathrm{CDCl}_{3}, 101 \mathrm{MHz}\right): \delta 173.8,155.7,140.0,135.2,128.3,126.2,71.43,71.38,65.2,56.0$, $55.1,50.8,43.9,43.8,42.6,40.3,39.9,39.4,37.5,37.1,35.6,35.0,34.2,33.4,31.8,30.4$, 28.8, 27.0, 23.5, 21.3, 18.6, 12.3. HRMS: exact mass calculated for $[\mathrm{M}+\mathrm{Na}]^{+}$ $\left(\mathrm{C}_{35} \mathrm{H}_{50} \mathrm{Cl}_{2} \mathrm{~N}_{2} \mathrm{NaO}_{5}\right)$ requires $671.2989 \mathrm{~m} / \mathrm{z}$, found $671.2957 \mathrm{~m} / \mathrm{z}$.

\section{3,5-Dichlorobenzyl}

(1-((4R)-4-((3R,7S,10S,13R)-3,7-dihydroxy-10,13dimethylhexadecahydro-1H-cyclopenta[a]phenanthren-17-yl)pentanoyl)azetidin-3-

yl)carbamate (16). Prepared according to General Procedure D with ursodeoxycholic acid (157 mg, $0.40 \mathrm{mmol}, 1.1$ equiv), $N, N$-diisopropylethylamine (314 $\mu \mathrm{L}, 1.8 \mathrm{mmol}, 4.0$ equiv), HATU (152 mg, 0.40 mmol, 1.1 equiv), 3,5-dichlorobenzyl azetidin-3-ylcarbamate (100 mg, $0.36 \mathrm{mmol}, 1.0$ equiv) and DMF (1 mL). The crude material was subjected to the purification outlined in the General Procedure (silica gel, $0-10 \% \mathrm{MeOH}$ in $\mathrm{CH}_{2} \mathrm{Cl}_{2}$ ) to afford the desired product as a clear solid (140 mg, $60 \%$ ). Umax (neat): 3400, 2933, 2865, 1708, 1637, 1438, $1449 \mathrm{~cm}^{-1} .{ }^{1} \mathrm{H}$ NMR $\left(\mathrm{CDCl}_{3}, 400 \mathrm{MHz}\right): \delta$ 7.33-7.29 (m, 1H), 7.23-7.19 (m, 2H), 5.63 (br. s, 1H), $5.04(\mathrm{~s}, 2 \mathrm{H}), 4.55-4.46(\mathrm{~m}, 1 \mathrm{H}), 4.41(\mathrm{t}, J=8.0 \mathrm{~Hz}, 1 \mathrm{H}), 4.29(\mathrm{t}, J=9.1 \mathrm{~Hz}, 1 \mathrm{H}), 3.97$ (dd, $J=5.1,8.5 \mathrm{~Hz}, 1 \mathrm{H}), 3.83(\mathrm{dd}, J=5.2,10.3 \mathrm{~Hz}, 1 \mathrm{H}), 3.64-3.52(\mathrm{~m}, 2 \mathrm{H}), 2.16-2.04(\mathrm{~m}$, 1H), 2.03-1.95 (m, 2H), 1.92-1.84 (m, 1H), 1.83-1.74 (m, 4H), 1.73-1.70 (m, 2H), 1.69-1.63 $(\mathrm{m}, 2 \mathrm{H}), 1.62-1.54(\mathrm{~m}, 2 \mathrm{H}), 1.53-1.38(\mathrm{~m}, 5 \mathrm{H}), 1.34-1.20(\mathrm{~m}, 5 \mathrm{H}), 1.16-0.99(\mathrm{~m}, 3 \mathrm{H}), 0.96-$ $0.88(\mathrm{~m}, 6 \mathrm{H}), 0.66(\mathrm{~s}, 3 \mathrm{H}) .{ }^{13} \mathrm{C}$ NMR $\left(\mathrm{CDCl}_{3}, 101 \mathrm{MHz}\right): \delta 174.1,169.1,139.7,135.3,128.5$, $126.3,71.6,71.5,65.4,58.1,55.9,55.1,54.9,51.0,43.9,42.6,40.9,40.3,39.3,37.5,37.0$, $35.6,35.1,34.2,31.0,30.5,28.8,28.7,27.0,23.5,21.3,18.7,12.3$. HRMS: exact mass calculated for $[\mathrm{M}-\mathrm{H}]^{-}\left(\mathrm{C}_{35} \mathrm{H}_{49} \mathrm{Cl}_{2} \mathrm{~N}_{2} \mathrm{O}_{5}\right)$ requires $647.3024 \mathrm{~m} / \mathrm{z}$, found $647.3021 \mathrm{~m} / \mathrm{z}$.

\section{3,5-Dichlorobenzyl}

((3R)-3-((4R)-4-((3R,7S,10S,13R)-3,7-dihydroxy-10,13dimethylhexadecahydro-1H-cyclopenta[a]phenanthren-17yl)pentanamido)cyclopentyl)carbamate (17). Prepared according to General Procedure E 
with ursodeoxycholic acid (149 mg, $0.38 \mathrm{mmol}, 1.1$ equiv), N,N-diisopropylethylamine (306 $\mu \mathrm{L}, 1.75 \mathrm{mmol}, 5.0$ equiv), HATU (145 mg, $0.38 \mathrm{mmol}, 1.1$ equiv), 3,5-dichlorobenzyl ((3R)3-aminocyclopentyl)carbamate (100 mg, $0.35 \mathrm{mmol}, 1.0$ equiv) and DMF (10 mL). The crude material was subjected to the purification outlined in the General Procedure (silica gel, 0$10 \% \mathrm{MeOH}$ in $\mathrm{CH}_{2} \mathrm{Cl}_{2}$ ) to afford the desired product as a clear solid (82 $\mathrm{mg}, 35 \%$ ). Umax (neat): 3301, 2931, 2968, 1697, 1654, $1420 \mathrm{~cm}^{-1} .{ }^{1} \mathrm{H}$ NMR $\left(\mathrm{CDCl}_{3}, 400 \mathrm{MHz}\right): \delta$ 7.29-7.26 (m, 1H), 7.22-7.18 (m, 2H), $5.03(\mathrm{~s}, 2 \mathrm{H}), 4.50-4.41(\mathrm{~m}, 1 \mathrm{H}), 3.72-3.60(\mathrm{~m}, 1 \mathrm{H}), 3.60-3.42(\mathrm{~m}, 4 \mathrm{H})$, 3.30-3.20 (m, 1H), 2.25-2.12 (m, 2H), 2.08-2.01 (m, 1H), 1.99-1.93 (m, 1H), 1.89-1.71 (m, $6 \mathrm{H}), 1.68-1.61(\mathrm{~m}, 2 \mathrm{H}), 1.58-1.53(\mathrm{~m}, 2 \mathrm{H}), 1.49-1.38(\mathrm{~m}, 6 \mathrm{H}), 1.31-1.19(\mathrm{~m}, 5 \mathrm{H}), 1.14-0.97$ $(\mathrm{m}, 3 \mathrm{H}), 0.96-0.87(\mathrm{~m}, 6 \mathrm{H}), 0.63(\mathrm{~s}, 3 \mathrm{H}) . \mathrm{NH}$ and $2 \times \mathrm{OH}$ not observed (exchangeable). ${ }^{13} \mathrm{C}$ $\operatorname{NMR}\left(\mathrm{CDCl}_{3}, 101 \mathrm{MHz}\right): \delta 173.8,154.4,140.2,135.1,128.2,126.2,71.31,71.26,65.4,56.0$, $55.1,51.6,49.4,48.6,44.6,44.2,43.8,43.7,42.6,40.3,39.4,37.4,37.2,35.5,35.0,34.1$, $33.5,31.9,30.9,30.4,28.8,27.0,23.5,21.3,18.6,12.2$. Rotameric mixture observed. HRMS: exact mass calculated for $\left[\mathrm{M}+\mathrm{NH}_{4}\right]^{+}\left(\mathrm{C}_{36} \mathrm{H}_{56} \mathrm{Cl}_{2} \mathrm{~N}_{3} \mathrm{O}_{5}\right)$ requires $680.3592 \mathrm{~m} / \mathrm{z}$, found $380.3588 \mathrm{~m} / \mathrm{z}$.

\section{3,5-Dichlorobenzyl}

((3S)-3-((4R)-4-((3R,7S,10S,13R)-3,7-dihydroxy-10,13-

\section{dimethylhexadecahydro-1H-cyclopenta[a]phenanthren-17-}

yl)pentanamido)cyclopentyl)carbamate (18). Prepared according to General Procedure E with ursodeoxycholic acid (200 mg, $0.51 \mathrm{mmol}, 1.1$ equiv), $\mathrm{N}, \mathrm{N}$-diisopropylethylamine (330 $\mu \mathrm{L}, 0.19 \mathrm{mmol}, 4.0$ equiv), HATU (194 mg, $0.51 \mathrm{mmol}, 1.1$ equiv), 3,5-dichlorobenzyl 3,5dichlorobenzyl (S)-3-aminopyrrolidine-1-carboxylate (136 mg, $0.47 \mathrm{mmol}, 1.0$ equiv) and DMF $(10 \mathrm{~mL})$. The crude material was subjected to the purification outlined in the General Procedure (silica gel, $0-10 \% \mathrm{MeOH}$ in $\mathrm{CH}_{2} \mathrm{Cl}_{2}$ ) to afford the desired product as a clear solid (54 mg, 17\%). U $U_{\max }$ (neat): 3450, 3415, 3030, 3963, 2940, 2862, 1725, $1637 \mathrm{~cm}^{-1} .{ }^{1} \mathrm{H}$ NMR $\left(\mathrm{CDCl}_{3}, 400 \mathrm{MHz}\right): \delta$ 7.32-7.29 (m, 1H), 7.25-7.21 (m, 2H), $5.91(\mathrm{~s}, 1 \mathrm{H}), 5.04(\mathrm{~s}, 2 \mathrm{H}), 4.46$ (m, 1H), 372-3.61 (m, 3H), 3.62-3.54 (m, 2H), 3.54-3.46 (m, 2H), $3.25(\mathrm{t}, J=12.1 \mathrm{~Hz}, 1 \mathrm{H})$, 
2.25-2.12 (m, 2H), 2.10-2.01 (m, 1H), 2.02-1.96 (m, 1H), 1.83-1.73 (m, 5H), 1.69-1. $62(\mathrm{~m}$, $2 \mathrm{H}), 1.60-1.53(\mathrm{~m}, 2 \mathrm{H}), 1.50-1.37(\mathrm{~m}, 5 \mathrm{H}), 1.25-1.18(\mathrm{~m}, 8 \mathrm{H}), 1.14-0.97(\mathrm{~m}, 3 \mathrm{H}), 0.96-0.86$ $(\mathrm{m}, 6 \mathrm{H}), 0.65(\mathrm{~s}, 3 \mathrm{H}) .{ }^{13} \mathrm{C} \mathrm{NMR}\left(\mathrm{CDCl}_{3}, 101 \mathrm{MHz}\right): \delta 173.7,154.5,140.2,135.2,128.3$, $126.2,71.4,65.4,58.5,55.9,55.1,51.7,49.5,48.7,44.6,44.2,43.9,42.6,40.3,39.4,38.7$, $37.4,37.1,35.5,35.0,34.2,33.5,32.0,31.8,30.9,30.4,28.8,27.0,23.5,21.3,18.6,18.5$, 12.2. Rotameric mixture observed. HRMS: exact mass calculated for $[\mathrm{M}+\mathrm{Na}]^{+}$ $\left(\mathrm{C}_{36} \mathrm{H}_{52} \mathrm{Cl}_{2} \mathrm{~N}_{2} \mathrm{NaO}_{5}\right)$ requires $685.3145 \mathrm{~m} / \mathrm{z}$, found $685.3129 \mathrm{~m} / \mathrm{z}$.

\section{3,5-Dichlorobenzyl}

((3R)-1-((4R)-4-((3R,7S,10S,13R)-3,7-dihydroxy-10,13dimethylhexadecahydro-1H-cyclopenta[a]phenanthren-17-yl)pentanoyl)pyrrolidin-3yl)carbamate (19). Prepared according to General Procedure E with ursodeoxycholic acid (149 mg, 0.38 mmol, 1.1 equiv), $N, N$-diisopropylethylamine (306 $\mu \mathrm{L}, 1.75 \mathrm{mmol}, 5.0$ equiv), HATU (145 mg, 0.38 mmol, 1.1 equiv), 3,5-dichlorobenzyl $(R)$-pyrrolidin-3-ylcarbamate (100 $\mathrm{mg}, 0.35 \mathrm{mmol}, 1.0$ equiv) and DMF (10 mL). The crude material was subjected to the purification outlined in the General Procedure (silica gel, $0-10 \% \mathrm{MeOH}$ in $\mathrm{CH}_{2} \mathrm{Cl}_{2}$ ) to afford the desired product as a clear solid (36 mg, 15\%). Umax (neat): 3298, 2933, 2868, 1706, 1624, $1436 \mathrm{~cm}^{-1} .{ }^{1} \mathrm{H}$ NMR $\left(\mathrm{CDCl}_{3}, 500 \mathrm{MHz}\right): \delta$ 7.33-7.30 (m, 1H), 7.24-7.21 (m, 2H), 5.07-5.02 (m, 2H), 5.02-4.97 (m, 1H), 4.36-4.21 (m, 1H), 3.78-3.65 (m, 1H), 3.62-3.50 (m, 4H), 3.45-3.31 $(\mathrm{m}, 1 \mathrm{H}), 2.33-2.22(\mathrm{~m}, 1 \mathrm{H}), 2.22-2.09(\mathrm{~m}, 1 \mathrm{H}), 2.04-1.97(\mathrm{~m}, 1 \mathrm{H}), 1.93-1.86(\mathrm{~m}, 1 \mathrm{H}), 1.85-$ $1.75(\mathrm{~m}, 4 \mathrm{H}), 1.71-1.63(\mathrm{~m}, 2 \mathrm{H}), 1.63-1.55(\mathrm{~m}, 4 \mathrm{H}), 1.53-1.47(\mathrm{~m}, 2 \mathrm{H}), 1.47-1.41(\mathrm{~m}, 4 \mathrm{H})$, 1.39-1.18 (m, 6H), 1.17-0.98 (m, 3H), 0.97-0.90 (m, 6H), $0.67(\mathrm{~s}, 3 \mathrm{H}) . \mathrm{NH}$ not observed. ${ }^{13} \mathrm{C}$ $\operatorname{NMR}\left(\mathrm{CDCl}_{3}, 101 \mathrm{MHz}\right): \delta 172.8,172.7,155.6,155.5,139.9,139.8,135.24,135.20,128.4$ $128.3,126.23,126.20,71.5,71.4,65.22,65.15,55.9,55.1,52.5,51.5,51.0,50.8,50.0,44.7$, $43.85,43.82,43.78,42.6,40.3,39.3,37.4,37.1,35.6,35.5,35.1,34.2,32.4,31.8,31.4$, $31.0,30.4,28.82,28.78,27.0,23.5,21.3,18.7,12.3$. Rotameric mixture observed. HRMS: exact mass calculated for $[\mathrm{M}+\mathrm{H}]^{+}\left(\mathrm{C}_{36} \mathrm{H}_{53} \mathrm{Cl}_{2} \mathrm{~N}_{2} \mathrm{O}_{5}\right)$ requires $663.3326 \mathrm{~m} / \mathrm{z}$, found 663.3323 $m / z$. 
yl)carbamate (20). Prepared according to General Procedure E with ursodeoxycholic acid (200 mg, $0.51 \mathrm{mmol}, 1.1$ equiv), $N, N$-diisopropylethylamine (330 $\mu \mathrm{L}, 0.19 \mathrm{mmol}, 4.0$ equiv), HATU (194 mg, 0.51 mmol, 1.1 equiv), 3,5-dichlorobenzyl 3,5-dichlorobenzyl (S)-pyrrolidin3-ylcarbamate (136 mg, $0.47 \mathrm{mmol}, 1.0$ equiv) and DMF (10 mL The crude material was subjected to the purification outlined in the General Procedure (silica gel, $0-10 \% \mathrm{MeOH}$ in $\mathrm{CH}_{2} \mathrm{Cl}_{2}$ ) to afford the desired product as a clear solid (56 mg, 18\%). Umax (neat): 3294,2931 , 2866, 1708, 1626, $1436 \mathrm{~cm}^{-1} .{ }^{1} \mathrm{H}$ NMR $\left(\mathrm{CDCl}_{3}, 500 \mathrm{MHz}\right): \delta$ 7.32-7.29 (m, 1H), 7.26-7.21 (m, 2H), 5.08-5.02 (m, 2H), 4.34-4.20 (m, 1H), 3.77-3.65 (m, 1H), 3.65-3.48 (m, 4H), 3.45-3.29 $(\mathrm{m}, 1 \mathrm{H}), 2.33-2.23(\mathrm{~m}, 1 \mathrm{H}), 2.20-2.11(\mathrm{~m}, 1 \mathrm{H}), 2.06-1.97(\mathrm{~m}, 1 \mathrm{H}), 1.95-1.87(\mathrm{~m}, 1 \mathrm{H}), 1.84-$ $1.74(\mathrm{~m}, 4 \mathrm{H}), 1.71-1.64(\mathrm{~m}, 2 \mathrm{H}), 1.62-1.53(\mathrm{~m}, 5 \mathrm{H}), 1.52-1.41(\mathrm{~m}, 6 \mathrm{H}), 1.37-1.30(\mathrm{~m}, 2 \mathrm{H})$, 1.30-1.21 (m, 4H), 1.17-1.11 (m, 1H), 1.09-0.98 (m, 2H), 0.97-0.91 (m, 6H), $0.67(\mathrm{~d}, J=2.0$ $\mathrm{Hz}, 3 \mathrm{H}) .{ }^{13} \mathrm{C} \mathrm{NMR}\left(\mathrm{CDCl}_{3}, 101 \mathrm{MHz}\right): \delta 172.8,172.7,155.4,139.8,139.7,135.3,128.5$, $126.3,71.6,71.5,65.3,55.9,55.1,52.6,51.5,51.1,50.0,44.6,43.9,43.8,42.6,40.3,39.3$, $37.5,37.0,35.6,35.1,34.2,32.5,31.8,31.4,31.0,30.5,29.8,28.8,27.1,23.5,21.3,18.8$, 12.3. Rotameric mixture observed. HRMS: exact mass calculated for $[\mathrm{M}+\mathrm{Na}]^{+}$ $\left(\mathrm{C}_{36} \mathrm{H}_{52} \mathrm{Cl}_{2} \mathrm{~N}_{2} \mathrm{NaO}_{5}\right)$ requires $685.3145 \mathrm{~m} / \mathrm{z}$, found $685.3113 \mathrm{~m} / \mathrm{z}$.

Protein production and crystallization. ATX from rat was produced in HEK293 Flp-In cells as described previously. ${ }^{16}$ For co-crystallization of rATX with $3, \mathbf{1 1}, \mathbf{5}$, and $\mathbf{1 7}$, rATX $(3.5 \mathrm{mg}$ $\mathrm{mL}^{-1}$ ) was first mixed in 10 molar excess with the compound and incubated for $30 \mathrm{~min}$. In all cases, the best crystals were obtained at room temperature $(293 \mathrm{~K})$ in a 24-well optimization screen (18-20\% PEG 3350, 0.1-0.4 M NaSCN and 0.1-0.4 $\mathrm{M} \mathrm{NH}_{4} \mathrm{l}$ ) by mixing $1 \mu \mathrm{L}$ of protein:compound solution and $1 \mu \mathrm{L}$ of reservoir solution. All crystals were vitrified in cryoprotectant, which consisted of reservoir solution with the addition of $20 \%(\mathrm{v} / \mathrm{v})$ glycerol. 
Crystallographic data and methods. The X-ray diffraction data for the ATX-inhibitor complexes were collected at SLS on beamlines PXIII (3, 11, and 5) and PXI (17) at 100K. The crystallographic diffraction data for the ATX-inhibitor complexes were recorded on a PILATUS $2 \mathrm{M}-\mathrm{F}$ detector for $\mathbf{3}, \mathbf{1 1}$, and $\mathbf{5}$ to a resolution of $1.95,2.4$, and $2.1 \AA$, respectively and on the EIGER $16 \mathrm{M}$ detector for 17 to $2.1 \AA$. All data were processed and integrated with XDS. ${ }^{17} 17$ was processed on site using the SLS automated processing pipeline and scaled with AIMLESS. ${ }^{18}$ The structures were determined by molecular replacement using PHASER ${ }^{19}$ with the structure of ATX (PDB entry 2XR9) as the search model. Model building and subsequent refinement were performed iteratively with $\mathrm{COOT}^{20}$ REFMAC5 ${ }^{21}$ and PDB_REDO. ${ }^{22}$ Structure validation was carried out by MolProbity. The structure models and experimental diffraction data were deposited at the PDB under codes 5MOS, 5MOM, 5MOD, 5MOE). Crystallographic data and refinement details are available in Table 2. 
Table 2. Crystallography Details

\begin{tabular}{|c|c|c|c|c|}
\hline & 3 & 5 & 11 & 17 \\
\hline \multicolumn{5}{|l|}{ Data Collection } \\
\hline Wavelength $(\AA)$ & 1.0000 & 0.9801 & 0.9801 & 1.0000 \\
\hline Resolution $(\AA)$ & 1.95 & 2.4 & 2.1 & 2.1 \\
\hline Space Group & P 1 & P 1211 & P 1211 & P 1211 \\
\hline $\begin{array}{l}\text { Unit Cell } a, b, c(\AA) \\
\alpha, \beta, \gamma\left({ }^{\circ}\right)\end{array}$ & $\begin{array}{c}53.6,61.7,63.7 \\
103,98,93\end{array}$ & $\begin{array}{c}62.9,88.0,77.4 \\
\alpha=\beta=\gamma=90\end{array}$ & $\begin{array}{c}62.8,88.9,77.3 \\
\alpha=\beta=\gamma=90\end{array}$ & $\begin{array}{c}62.9,89.3,77.2 \\
\alpha=\beta=\gamma=90\end{array}$ \\
\hline $\mathrm{CC}_{1 / 2}$ & $0.996(0.481)$ & $0.998(0.677)$ & $0.998(0.568)$ & $0.998(0.487)$ \\
\hline$R_{\text {merge }}$ & $0.044(0.812)$ & $0.025(0.921)$ & $0.027(1.148)$ & $0.026(0.766)$ \\
\hline$|/ \sigma|$ & $18.9(1.5)$ & $41.9(1.6)$ & $32.6(1.2)$ & $19.1(1.6)$ \\
\hline Completeness (\%) & $98.8(96.6)$ & $98.3(99.6)$ & $97.1(97.9)$ & $89.2(95.4)$ \\
\hline Redundancy & $3.9(3.8)$ & $4.5(4.5)$ & $3.8(4.0)$ & $27(2.5)$ \\
\hline \multicolumn{5}{|l|}{ Refinement } \\
\hline No. atoms & 6700 & 6451 & 6592 & 6598 \\
\hline Protein & 6275 & 6251 & 6275 & 6242 \\
\hline Ligand/Metal/Glycan & 186 & 172 & 215 & 256 \\
\hline Water/lodine & 239 & 28 & 102 & 100 \\
\hline TLS groups & 1 & 1 & 1 & 1 \\
\hline$R_{\text {work }} / R_{\text {free }}(\%)$ & $18.1 / 22.3$ & $22.4 / 27.0$ & $23.8 / 28.6$ & $20.1 / 24.9$ \\
\hline \multicolumn{5}{|l|}{ Validation } \\
\hline Rmsd/rmsZ bond lengths $(\AA)$ & 0.012 & 0.008 & 0.007 & 0.011 \\
\hline Rmsd/rmsZ bond angles $\left({ }^{\circ}\right)$ & 1.631 & 1.377 & 1.249 & 1.515 \\
\hline $\begin{array}{l}\text { Ramachandran } \\
\text { Preferred/ outliers }\end{array}$ & $98 / 0$ & $95 / 0$ & $97 / 0$ & $96 / 0$ \\
\hline Sidechain rotamers Preferred & 94 & 94 & 96 & 95 \\
\hline MolProbity/Clash sco & $100 / 100$ & $100 / 100$ & $100 / 100$ & $100 / 100$ \\
\hline
\end{tabular}

High Resolution shell in parentheses 
Biochemical assays and modeling of kinetic data. ATX lysoPLD activity was measured by choline release from LPC. ${ }^{23} 20 \mathrm{nM}$ human ATX (prepared from HEK $293 \mathrm{Flp}$-In cells) was incubated $150 \mathrm{mM}$ LPC (18:1) in a final volume of $100 \mu \mathrm{L}$ buffer containing $50 \mathrm{mM}$ Tris, $\mathrm{pH}$ 7.4, $0.01 \%$ Triton $\mathrm{X}-100,50 \mathrm{mM} \mathrm{CaCl}_{2}, 1 \mathrm{U} \mathrm{mL}^{-1}$ horseradish peroxidase, $2 \mathrm{mM}$ homovanilic acid (HVA). The relative amount of released choline was measured by HVA fluorescence in a 96-well plate (Corning). Fluorescence intensity was determined at ex/em 320/450 nm every $30 \mathrm{~s}$ for 90 min with a Fluorostart plate reader (BMG Labtech). Data analysis was performed using GraphPad Prism. The experiments for determining $\mathrm{IC}_{50}$ values for the different inhibitors were performed by serial threefold dilutions; for each inhibitor concentration, the velocity of the reaction was monitored as a function of time in the lysoPLD assay as described above. The linear part of the reaction velocity was visually estimated (typically between $15 \mathrm{~min}$ and $60 \mathrm{~min}$ ) and the observed fluorescence signal (described above) as a function of time was modeled for all inhibitor concentrations simultaneously using the formula:

$$
F_{t}=F_{0}+\left(\frac{v_{\max }-v_{\min }}{\left(1+C_{i n h} / I C_{50}\right)}+v_{\min }\right) t
$$

where Ft is the observed fluorescence signal relative to the choline product at each measurement, $v_{\max }$ and $v_{\min }$ are the fitted values for the minimum and maximum relative velocity and $c_{i n h}$ is the corresponding inhibitor concentration. To identify the mode of inhibition of ATX by 17 , we used regression analysis tools within the GraphPad Prism software to determine the value of $\alpha$ using the built-in formula for the mixed inhibition model. The values of all derived parameters indicate mean values as obtained by nonlinear regression analysis; triplicate data were used as separate observations during the fit; \pm values indicate the standard error of the mean.

Cell-based assays. BJEH fibroblasts were seeded in 6-well tissue culture plates in DMEM (GIBCO, Life Technologies) containing 10\% FCS (Hyclone, Thermo Scientific), 2 mM glutamine, $100 \mathrm{UmL}^{-1}$ penicillin and $100 \mathrm{mgmL}^{-1}$ streptomycin (GIBCO, Life Technologies). When \pm 60 -90 confluent, they were serum starved (DMEM with glutamine and pen/strep, 
without FCS) for 16 hours. hATX (10 nM final concentration) was incubated with inhibitor for $30 \mathrm{~min}$ in serum free medium (total volume $1 \mathrm{~mL}$ ). Medium from the 6-well plates was removed and replaced with $1 \mathrm{~mL}$ of ATX-inhibitor (17) mixture. Cells were stimulated for 15 min, medium was removed and plates were immediately frozen on dry ice and stored at $80{ }^{\circ} \mathrm{C}$. Cells were lysed in the plates on ice for $\pm 10 \mathrm{~min}$ (lysis buffer: RIPA, containing sodium fluoride, orthovanadate and protease inhibitors). Lysates were centrifuged for 30 minutes at $10.000 \mathrm{rpm}$ to remove cell debris and were taken up in loading buffer containing DTT and boiled for $10 \mathrm{~min}$ at $95^{\circ} \mathrm{C}$. Samples were loaded on a pre-cast gel (Bis-Tris Invitrogen 4-12\%) and separated by gel electrophoresis. The gel was transferred by western blotting to a nitrocellulose membrane which was blocked in milk (to reduce non-specific binding) before the membrane was probed with the desired primary antibodies overnight (in this case p-AKT and total AKT). The membrane was washed $3 \times$ in PBS-tween and probed with a HRP-conjugated anti-Rabbit antibody for 1 hour. Washed $3 \times$ and developed using ECL reagent using a BioRad chemidoc imaging system.

Mouse experiments. ATX inhibitors were dissolved at $50 \mathrm{mgmL}^{-1}$ in vehicle (Hydroxypropyl Cellulose $2 \%$ / Tween 80 0.1\%) and administered intraperitoneally at $10 \mathrm{mgkg}^{-1}$. Blood was collected after 8 hours, plasma isolated and levels of 8 abundant LPA species determined by HPLC electrospray ionization tandem mass spectrometry as reported previously. ${ }^{15}$ Protocols and procedures for animal studies conformed to current US Public Health Service policy and were reviewed and approved by the Institutional Animal Care and Use Committee at the University of Kentucky.

\section{Abbreviations}

Autotaxin (ATX); Human transformed skin fibroblasts (BJEH); Ectonucleotide pyrophosphatase 2 (ENPP2); Lysophosphatidic acid (LPA); Lysophosphatidyl choline (LPC); phosphor-serine/threonine protein kinase (pAKT); Structure activity relationship (SAR); Somatomedin- $\beta$ (SMB); Tauroursodeoxycholic acid (TUDCA); Ursodeoxycholic acid (UDCA). 


\section{Associated Content}

Supporting information. Spectral data for all compounds, preparative details for diamine monomers and crystallographic refinement data (PDF).

\section{Author Information}

‘Corresponding authors a.perrakis@nki.nl; craig.jamieson@strath.ac.uk

Telephone: +44 1415484830 (CJ); +31205121951 (AP).

\section{Acknowledgements}

For financial support we thank: NWO for supporting work on Autotaxin (700.10.354) for support to the lab of AP; GlaxoSmithKline, EPRSC (EP/L505080/1) and Scottish Funding Council for a PhD studentship for FP; NIH (1R01HL120507), VA (BX001984-01), DOD (BC150305P1) for support to the University of Kentucky teams. High resolution mass spectral data was obtained at the EPSRC Mass Spectrometry facility at the University of Swansea, UK.

\section{Accession Codes}

PDB codes for the structures presented are 5MOE (3), 5MOS (5), 5MOD (11) and 5MOM (17). Authors will release the atomic coordinates and experimental data upon article publication. 


\section{References}

1. Moolenaar, W. H.; Perrakis, A. Insights into Autotaxin: How to Produce and Present a Lipid Mediator. Nat. Rev. Mol. Cell Biol. 2011, 12, 674-679.

2. Choi, J. W.; Herr, D. R.; Noguchi, K.; Yung, Y. C.; Lee, C. W.; Mutoh, T.; Lin, M. E.; Teo,

S. T.; Park, K. E.; Mosley, A. N.; Chun, J. LPA Receptors: Subtypes and Biological Actions. Annu. Rev. Pharmacol. Toxicol. 2010, 50, 157-186.

3. Castagna, D.; Budd, D. C.; Macdonald, S. J. F.; Jamieson, C.; Watson, A. J. B. Development of Autotaxin Inhibitors, An Overview of the Patent and Primary Literature. J. Med. Chem. 2016, 59, 5604-5621.

4. Hausmann, J.; Kamtekar, S.; Christodoulou, E.; Day, J. E.; Wu, T.; Fulkerson, Z.; Albers, H. M.; van Meeteren, L. A.; Houben, A. J.; van Zeijl, L.; Jansen, S.; Andries, M.; Hall, T.; Pegg, L. E.; Benson, T. E.; Kasiem, M.; Harlos, K.; Kooi, C. W.; Smyth, S. S.; Ovaa, H.; Bollen, M.; Morris, A. J.; Moolenaar, W. H.; Perrakis A. Structural Basis of Substrate Discrimination and Integrin Binding by Autotaxin. Nat. Struct. Mol. Biol. 2011, 18, 198-204.

5. Nishimasu, H.; Okudaira, S.; Hama, K.; Mihara, E.; Dohmae, N.; Inoue, A.; Ishitani, R.; Aoki, J.; Nureki O. Crystal Structure of Autotaxin and Insight into GPCR Activation by Lipid Mediators. Nat. Struct. Mol. Biol. 2011, 18, 205-212.

6. Keune, W.-J.; Hausmann, J.; Bolier, R.; Tolenaars, D.; Kremer, A.; Heidebrecht, T.; Joosten, R. P.; Sunkara, M.; Morris, A. J.; Matas-Rico, E.; Moolenaar, W. H.; Elferink, R. P. O.; Perrakis, A. Steroid Binding to Autotaxin Links Bile Salts and Lysophosphatidic acid Signalling. Nat. Commun. 2016, 7, 11248. 
7. Stein, A. J.; Bain, G.; Prodanovich, P.; Santini, A. M.; Darlington, J.; Stelzer, N. M. P.; Sidhu, R. S.; Schaub, J.; Goulet, L.; Lonergan, D.; Calderon, I.; Evans, J. F.; Hutchinson, J. H. Structural Basis for Inhibition of Human Autotaxin by Four Potent Compounds with Distinct Modes of Binding. Mol. Pharmacol. 2015, 88, 982-992.

8. Shah, P.; Cheasty, A.; Foxton, C.; Raynham, T.; Faroog, M.; Gutierrez, I. F.; Lejeune, A.; Pritchard, M.; Turnbull, A.; Pang, L.; Owen, P.; Boyd, S.; Stowell, A.; Jordan, A.; Hamilton, N. M.; Hitchin, J. R.; Stockley, M.; MacDonald, E.; Quesada, M J.; Trivier, E.; Skeete, J.; Ovaa, H.; Moolenaar, W. H.; Ryder H. Discovery of Potent Inhibitors of the Lysophospholipase Autotaxin. Bioorg. Med. Chem. Lett. 2016, 26, 5403-5410.

9. Schiemann, K.; Schultz, M.; Blaukat, A.; Kober, I. Merck Patent Gmbh. Piperidine and Piperazine Derivatives, US 8754097 B2, 2014.

10. Gierse, J.; Thorarensen, A.; Beltey, K.; Bradshaw-Pierce, E.; Cortes-Burgos, L.; Hall, T.; Johnston, A.; Murphy, M.; Nemirovskiy, O.; Ogawa, S.; Pegg, L.; Pelc, M.; Prinsen, M.; Schnute, M.; Wendling, J.; Wene, S.; Weinberg, R.; Wittwer, A.; Zweifel, B.; Masferrer, J. A Novel Autotaxin Inhibitor Reduces Lysophosphatidic Acid Levels in Plasma and the Site of Inflammation. J. Pharmacol. Exp. Ther. 2010, 334, 310-317.

11. St-Cœur, P.-D.; Ferguson, D.; Morin, P. Jr.; Touaibia, M. PF-8380 and Closely Related Analogs: Synthesis and Structure-activity Relationship Towards Autotaxin Inhibition and Glioma Cell Viability. Arch. Pharm. 2013, 346, 91-97.

12. Jones, S. B.; Pfeifer, L. A.; Bleisch, T. J.; Beauchamp, T. J.; Durbin, J. D.; Klimkowski, V. J.; Hughes, N. E.; Rito, C. J.; Dao, Y.; Gruber, J. M.; Bai, H.; Chambers, M. G.; Chandrasekhar, S.; Lin, C.; McCann, D. J.; Mudra, D. R.; Oskins, J. L.; Swearingen, C. A.; Thirunavukkarasu, K.; Norman, B. H. Novel Autotaxin Inhibitors for the Treatment of 
Osteoarthritis Pain: Lead Optimization via Structure-Based Drug Design. ACS Med. Chem. Lett. 2016, 7, 857-861.

13. Kawaguchi, M.; Okabe, T.; Okudaira, S.; Nishimasu, H.; Ishitani, R.; Kojima, H.; Nureki, O.; Aoki, J.; Nagano, T. Screening and X-ray Crystal Structure-based Optimization of Autotaxin (ENPP2) Inhibitors, Using a Newly Developed Fluorescence Probe. ACS Chem. Biol. 2013, 8, 1713-1721.

14. JChem for Office (Excel) was used for, structure based property calculation: JChem for Office v15.6.29, ChemAxon (http://www.chemaxon.com), 2016.

15. Salous, A. K.; Panchatcharam, M.; Sunkara, M.; Mueller, P.; Dong, A.; Wang, Y.; Graf, G. A.; Smyth, S. S.; Morris, A. J. Mechanism of Rapid Elimination of Lysophosphatidic Acid and Related Lipids from the Circulation of Mice. J. Lipid Res. 2013, 54, 2775-2784.

16. Day, J. E.; Hall, T.; Pegg, L. E.; Benson, T. E.; Hausmann, J.; Kamtekar, S. Crystallization and Preliminary X-ray Diffraction Analysis of Rat Autotaxin. Acta Crystallogr. Sect. F Struct. Biol. Cryst. Commun. 2010, 66, 1127-1129.

17. Kabsch, W. Integration, Scaling, Space-group Assignment and Post-refinement. Acta Crystallogr. D Biol. Crystallogr. 2010, 66, 133-134.

18. Evans, P. R. An Introduction to Data Reduction: Space-group Determination, Scaling and Intensity Statistics. Acta Crystallogr. D Biol. Crystallogr. 2011, 67, 282-292.

19. McCoy, A. J. Solving Structures of Protein Complexes by Molecular Replacement with Phaser. Acta Crystallogr. D Biol. Crystallogr. 2007, 63, 32-41. 
20. Emsley, P.; Lohkamp, B.; Scott, W. G.; Cowtan, K. Features and Development of Coot. Acta Crystallogr. D Biol. Crystallogr. 2010, 66, 486-501.

21. Murshudov, G. N.; Skubák, P.; Lebedev, A. A.; Pannu, N. S.; Steiner, R. A.; Nicholls, R. A.; Winn, M. D.; Long, F.; Vagin, A. A. REFMAC5 for the Refinement of Macromolecular Crystal Structures. Acta Crystallogr. D Biol. Crystallogr. 2011, 67, 355-367.

22. Joosten, R. P.; Long, F.; Murshudov, G. N.; Perrakis, A. The PDB_REDO Server for Macromolecular Structure Model Optimization. IUCrJ 2014, 1, 213-220.

23. Albers, H. M. H. G.; Dong, A.; van Meeteren, L. A.; Egan, D. A.; Sunkara, M.; van Tilburg, E. W.; Schuurman, K.; van Tellingen, O.; Morris, A. J.; Smyth, S. S.; Moolenaar, W. H.; Ovaa, H. Boronic Acid-based Inhibitor of Autotaxin Reveals Rapid Turnover of LPA in the Circulation. Proc. Natl. Acad. Sci. U.S.A 2010, 107, 7257-7262. 
Table of Contents graphic

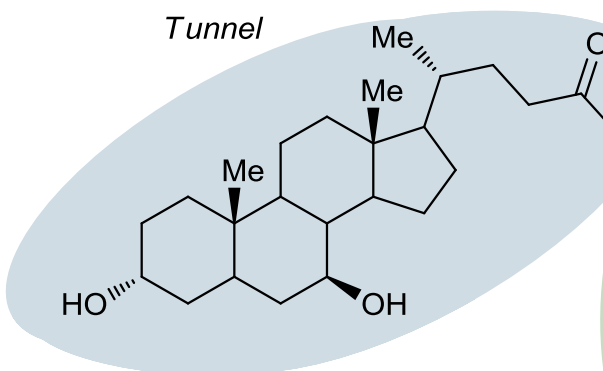

- competitive ATX inhibitor (nM)

$\mathrm{Cl}^{\prime}$<smiles>O=C(OCc1cc(Cl)cc(Cl)c1)N1CC[C@@H](NCO)C1</smiles>

- identified through structure based design

- modulates LPA synthesis in vivo

Lipophilic pocket 